Abstract Currently operating laser interferometric gravitational wave detectors are limited by quantum noise above a few hundred Hertz. Detectors that will come on line in the next decade are predicted to be limited by quantum noise over their entire useful frequency band (from $10 \mathrm{~Hz}$ to $10 \mathrm{kHz}$ ). Further sensitivity improvements will, therefore, rely on using quantum optical techniques such as squeezed state injection and quantum nondemolition, which will, in turn, drive these massive mechanical systems into quantum states. This article reviews the principles behind these optical and quantum optical techniques and progress toward there realization.

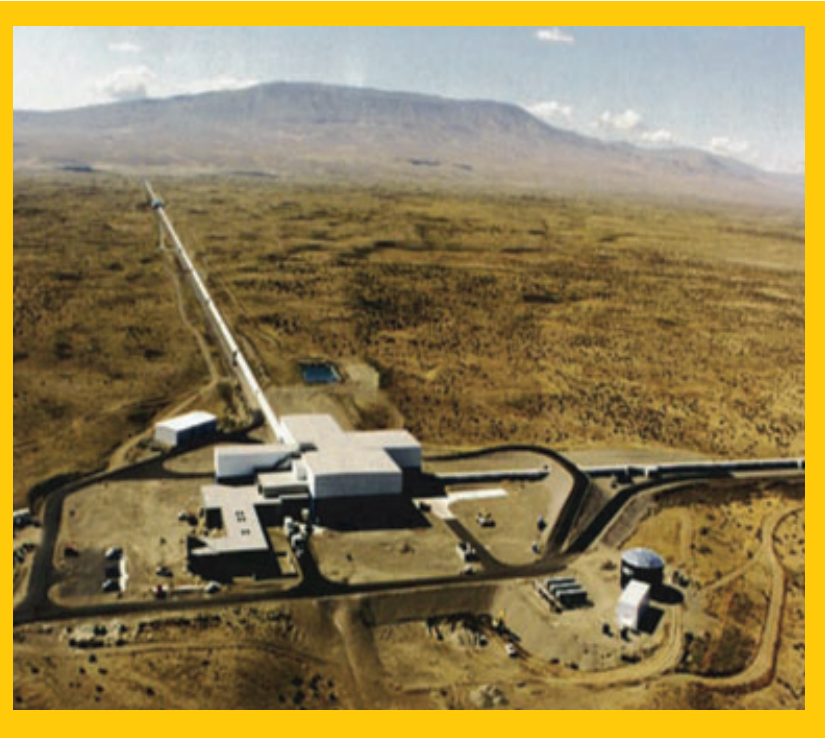

\title{
Advanced interferometry, quantum optics and optomechanics in gravitational wave detectors
}

\author{
David E. McClelland ${ }^{1,{ }^{*},}$ Nergis Mavalvala ${ }^{2}$, Yanbei Chen ${ }^{3}$, and Roman Schnabel ${ }^{4}$
}

\section{Introduction}

According to Einstein's general theory of relativity [1], the geometry of the space-time continuum is dynamical, and interacts with matter. Non-axisymmetric acceleration of matter creates oscillations of space-time geometry in their vicinity, which propagate away at the speed of light, causing "ripples" even at distant regions of space. These ripples are called gravitational waves. Conservation of mass and linear momentum dictates that gravitational radiation starts at mass quadrupole order, in much the same way as charge conservation dictates that electromagnetic radiation starts at charge dipole order. In other words, a graviton has a spin of 2 , while a photon has a spin of 1 .

Depending on the choice of coordinate systems, the physical effect of a gravitational wave on an array of freely falling test objects, with spatial separation less than its reduced wavelength $\lambda_{\mathrm{GW}} /(2 \pi)$, and light propagating between them can either be described by (i) a modulation of the refractive index of space alone, or (ii) solely by a tidal force field applied on the test masses. Similar to electromagnetic waves, a plane gravitational wave also has two polarizations (due to the fact that gravitons are massless), often denoted as + and $\times$. Taking description (ii) above, a plane gravitational wave propagating along the $z$ axis dis- torts a ring of free test objects on the $x$-y plane following patterns indicated by Fig. 1. More quantitatively, we have

$$
\delta \ddot{x}=\frac{x}{2} \ddot{h}_{+}+\frac{y}{2} \ddot{h}_{\times}, \quad \delta \ddot{y}=-\frac{y}{2} \ddot{h}_{+}+\frac{x}{2} \ddot{h}_{\times},
$$

where $h_{+}$and $h_{\times}$are amplitudes of the gravitational wave in + and $\times$ polarizations, $(x, y)$ denote a test object's unperturbed position on the phase front before the gravita-

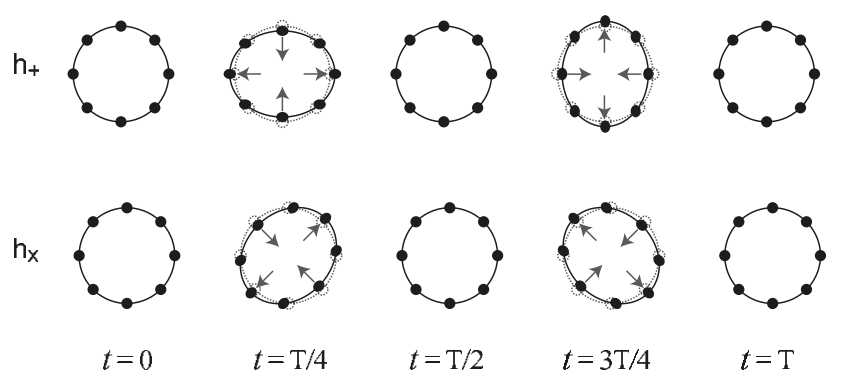

Figure 1 The effect of a gravitational wave propagating into the plane on a small ring of free test masses over a full gravitational wave period T. The top series of diagrams shows the effect of a gravitational wave in the $h_{+}$polarisation. The bottom series of diagrams shows the effect of a gravitational wave in the $h_{\times}$polarisation.

\footnotetext{
${ }^{1}$ Centre for Gravitational Physics, The Australian National University, Canberra, 0200, Australia ${ }^{2}$ LIGO Laboratory, Masachusetts Institute of Technology, Cambridge, MA 02139, USA ${ }^{3}$ LIGO, California Institute of Technology, Pasadena, CA 91109, USA ${ }^{4}$ Albert Einstein Institute, Leibniz Universität Hannover, Hannover, Germany
}

* Corresponding author: e-mail: David.McClelland@anu.edu.au 


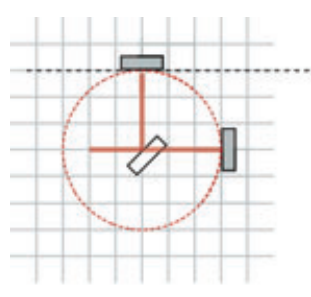

(a)

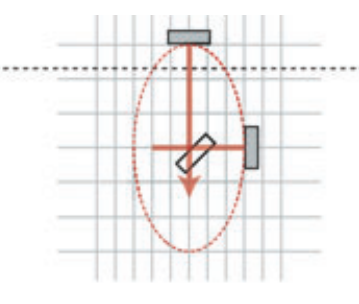

(b)

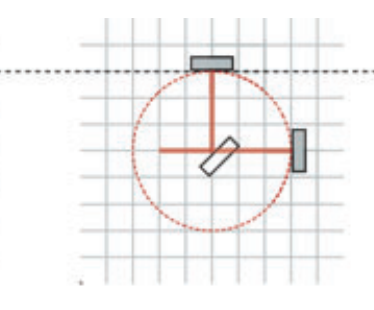

(c)

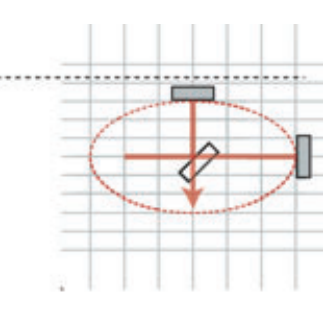

(d)

Figure 2 (online color at: www.lpr-journal.org) The effect of a gravitational wave propagating into the plane (in the $h_{+}$polarisation) on a Michelson interferometer.

tional wave arrives, $(\delta x, \delta y)$ the displacement caused by the gravitational wave, and double dots denote double time derivatives. Note that masses of the objects drop out, as a consequence of the Equivalence Principle.

The stiffness of space-time is enormous, even the most violent events in the universe produce waves with incredibly small strain amplitudes. One can appreciate this through calculating the energy flux of a linearly polarized plane gravitational wave:

$$
\frac{d E}{d A d t}=\frac{c^{3}}{16 \pi G}\left\langle\dot{h}^{2}\right\rangle
$$

where $\langle\ldots\rangle$ denotes averaging over a period of the wave, $c=3 \times 10^{8} \mathrm{~m} / \mathrm{s}$ is the speed of light, and $G=6.67 \times$ $10^{-11} \mathrm{~m}^{3} \mathrm{~kg}^{-1} \mathrm{~s}^{-2}$ is the Newton's constant of gravity. As an example, events such as a supernova in our galaxy or the collision of neutron stars within the local galaxy group will generate waves with amplitudes of the order $h \sim 10^{-21}$, yet their instantaneous energy flux in gravitational waves (assuming a frequency of $2 \mathrm{kHz}$ ) can reach $\sim 60 \mathrm{~W} / \mathrm{m}^{2}$, which is slightly higher than the electromagnetic energy flux under a reading light.

Early attempts to detect gravitational waves searched for gravitational wave (GW) induced excitations in the mechanical modes of solid bars of metal such as Aluminium or Niobium, culminating in detectors reaching sensitivities on the order of $10^{-19}$ in a very narrow band of frequencies centered about $\sim 1 \mathrm{kHz}$ [2]. Following the advent of the laser in 1959 and the progress in laser interferometry, Weiss [3] and separately Forward [4] proposed a gravitational wave detector based on the Michelson interferometer (Fig. 2). A passing gravitational wave will alternately stretch one interferometer arm whilst contracting the other arm (see Fig. 1) differentially changing the phase of the light beams in the 2 arms. The phase change and hence GW signal is read out on the interference pattern observed at the output port. Some 30 years later, the Michelson interferometer, which had such a profound impact on the development of special relativity in the 19th century, is now poised to prove the final prediction of Einstein's theory of general relativity and open an entirely new field of astronomy.

In order to reach the required sensitivities, all environmental perturbations to the interferometer mirrors (test masses) must be a significant factor smaller than the gravitational wave signal. Since the gravitational wave signal is related to the relative displacement of mirrors with separation $L$ by $\delta x \propto \delta h L$, the mirror separation is made as large as feasible to give the largest possible strain measurement. With ground based detectors, the test masses must be hung from sophisticated isolation systems to eliminate the background seismic noise and in an ultra high vacuum system to avoid refractive index fluctuations mimicking a signal. Great effort is required to minimize electronics noise and to avoid mirror feedback and control systems re-injecting noise onto the signal. Classical intensity and frequency noise on the laser beam has to be suppressed, aided by the common mode rejection of a Michelson interferometer operating on a dark fringe. Once all such technical noise has been made negligible, three 'fundamental' noise sources remain: (1) direct Newtonian coupling between the suspended test mass and the local gravity environment sets the low frequency limit to the performance of terrestrial detectors; (2) thermal noise arising from the Brownian motion in a mechanical system at non-zero temperature, which is typically distributed amongst mirror vibrational modes; suspension modes and coating modes; and (3) quantum noise arising from vacuum fluctuations in the electromagnetic field modes used to continuously sense and readout the position of nearly free test masses.

Fig. 3 shows the optical configuration an Fig. 4 the predicted sensitivity of the US Advanced LIGO [5] detectors due to come on line in 2014. The target displacement noise level of Advanced LIGO (aLIGO) is at a remarkable $4 \times 10^{-23} / \sqrt{\mathrm{Hz}}$ in its most sensitive band. With this performance, gravitational waves should be regularly detected. However, in order to reach cosmological distance scales and be able to probe the beginnings of the universe, even this

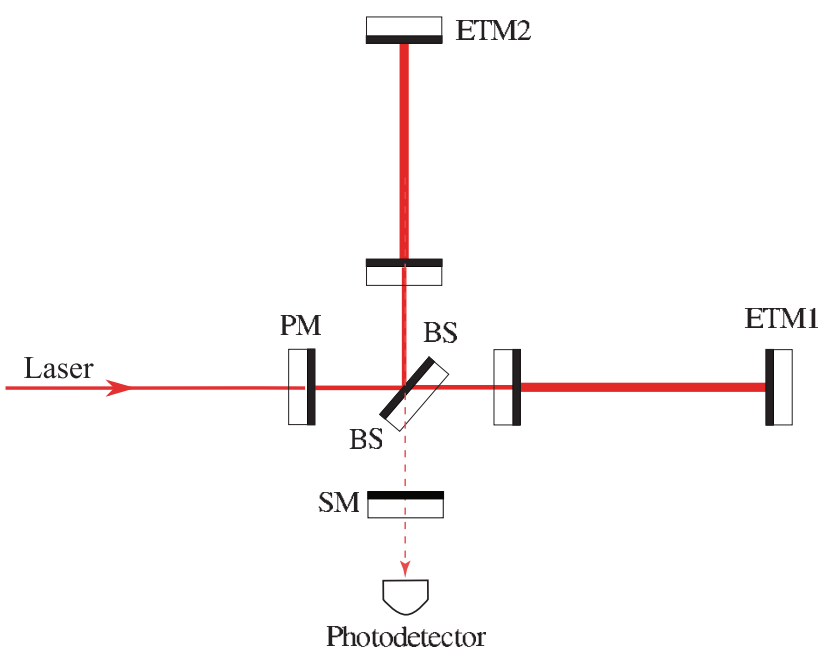

Figure 3 (online color at: www.lpr-journal.org) Schematic of the optical layout of second generation detectors such as Advanced LIGO. PM: power recycling mirror; SM: signal recycling mirror. 


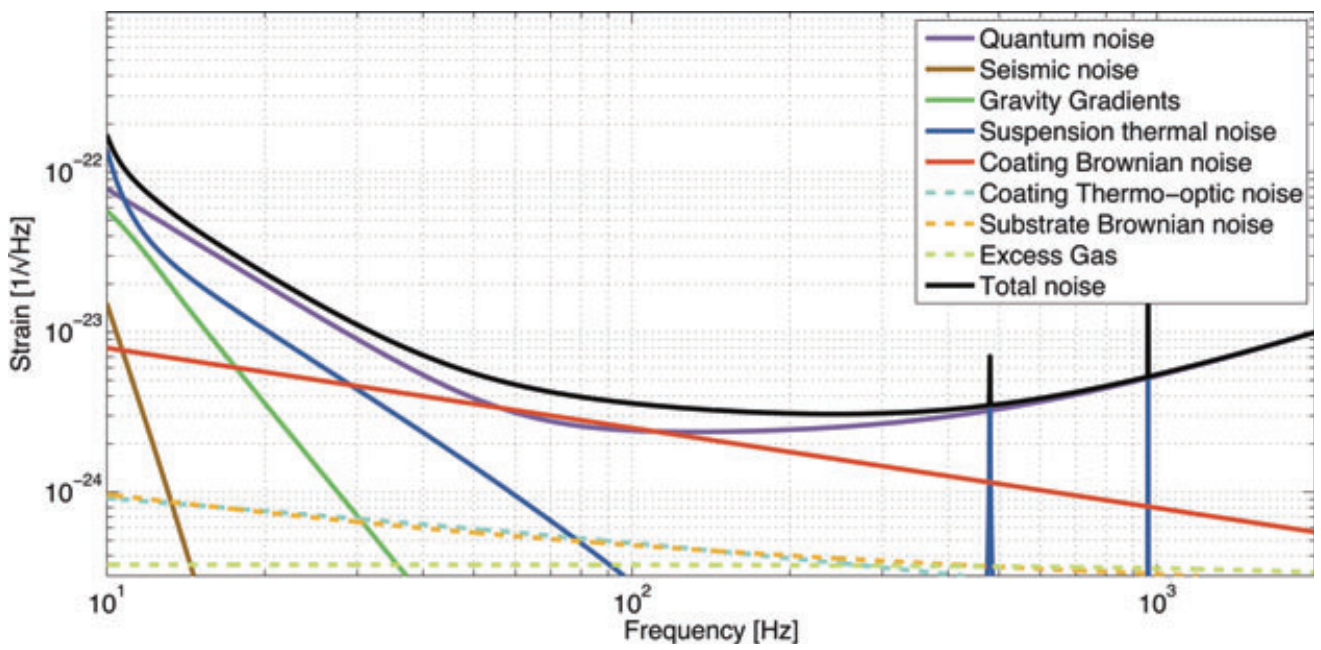

Figure 4 (online color at: www.lpr-journal.org) Predicted sensitivity of Advanced LIGO operating in the 'broadband' mode. sensitivity is not good enough; third-generation detectors with yet another factor of 10 improvement are currently being conceived.

Apart from a small frequency region around $100 \mathrm{~Hz}$, where thermal noise dominates, second generation detectors such as aLIGO should be limited by quantum noise across their entire frequency band from few $\mathrm{Hz}$ to few $\mathrm{kHz}$. Moreover, they will operate very close to the so-called Standard Quantum Limit [6], where the Heisenberg Uncertainty Principle constrains the quantum-noise-limited sensitivity of continuous measurements of the position of the $40 \mathrm{~kg}$ test mass. This quantum noise is the focus of the remainder of this article.

We begin with a brief overview of a gravitational wave detector as a gravito-optomechanical transducer in Sect. 2, before introducing the quantum nature of light and how it influences the measurement in Sect. 3. This leads to the concepts of shot noise, quantum radiation pressure noise, the standard quantum limit, squeezing and quantum non demolition (QND). In Sects. 4 and 5, we revisit the gravito-optomechanical transducer to consider how motion of the optomechanical oscillator can modify optical quantum noise.

Section 5 briefly re-examines the physics from the viewpoint of modes of a massive optomechanical oscillator instead of sensitivity to gravitational waves. Radiation pressure (RP) plays an important role not only in advanced GW detectors, but in other optomechanical systems that use the coupling between the mirror position and the radiation field for cavity-assisted cooling [7-12,14], to modify the dynamics of the interferometer mirrors [16-25], and to produce squeezing due to correlations between the shot noise and radiation pressure noise $[19,20]$. The mirror oscillator is the suspended mirror pendulum of GW detectors [24-26] and related experiments $[11,27]$, or nano- and micro-cantilevers and membranes $[7,8,10,12-15]$. The key ingredients of a system where radiation pressure dominates the dynamics are: relatively high stored optical power that impinges on a mirror oscillator that can respond to radiation pressure; mirror oscillators with high mechanical quality factors $(Q)$, to mitigate thermal noise and provide long coherence times; a low noise readout scheme to measure the mirror displacement; and sufficiently strong optomechanical coupling between the mirror and radiation field. This is the realm of optical cooling in which oscillator motion approaches its quantum ground state - macroscopic quantum mechanics on a truly grand scale.

We end with a review of the experimental achievements relevant to gravitational wave detectors to date and the near term outlook.

\section{GW detectors - the optical response}

As in any instrument a key design requirement is to maximize the signal to noise ratio. This is what determines the instrument's sensitivity. In this section we review interferometer configurations from the viewpoint of optimizing the signal response/bandwidth combination and then address the issue of minimizing quantum noise.

For illustrative purpose we consider the interferometer configuration depicted in Fig. 3. The central element is the optical cavity formed between mirrors that are hung from pendulum suspension systems. Well above the suspension resonance frequency the mirrors can be considered to respond freely to changes in their separation, in particular those arising from the passage of gravitational waves. Laser light enters the cavity and undergoes multiple reflections off the cavity mirrors sampling mirror motion each time, before leaking out to contribute to the total field reflected by the cavity. Thus the motion is read out as the change in phase of the total reflected field. In the frequency domain, the mirror motion transfers energy out of the carrier field, into phase modulation sidebands, called the signal sidebands. Due to the fact that gravitational wave induced motion reverses direction after half a period, on light stored for longer than this the induced phase will diminish. This sets the bandwidth of the detector. It is determined by the cavity length and the effective reflectivities of the mirrors. Typically, ground based detectors are a few kilometers long and operate in the acoustic signal frequency regime from $10 \mathrm{~Hz}$ to a few $\mathrm{kHz}$ and so the bandwidth is often selected to be about $100 \mathrm{~Hz}$. 
With a single cavity the GW signal will be swamped by laser noise. By inserting a cavity in each of the orthogonal arms of a Michelson Interferometer (MI) and operating the interferometer on a dark fringe, laser noise and any noise inducing signals common to both cavities will be suppressed by the common mode rejection factor (related to the fringe visibility, and typically of order $1 / 1000$ for the LIGO interferometers). However, due to their quadrupolar nature, the GWs act differentially on the two arms. Thus, at the antisymmetric port of the interferometer, the GW signal coming from the arms of the MI add coherently.

A further benefit of dark fringe operation is that, from the view point of the incident light, the MI appears to be a mirror, reflecting most of the light back on itself. By inserting a so called 'power recycling' mirror [28] this light can be re-used, interferometrically building up the power incident on the beam splitter (and therefore the cavities) and thus enhancing interferometer response.

This configuration of a power recycled, arm cavity Michelson interferometer is the layout adopted in the first generation of multi kilometer GW detectors (LIGO and Virgo). In the absence of noise, signal response is peaked around the carrier frequency and rolls off with the arm cavity bandwidth. The only way to increase the signal response is to increase the laser power. For a review of progress in the area see Willke [29]. In 1988, Meers [30] realised that the signal response could be tailored by placing a mirror (SM in Fig. 3) at the output port (dark fringe) of the Michelson interferometer to form a cavity which could resonate the GW induced signal sidebands. This process was called signal recycling. Sample tuning curves are shown in Fig. 5. There are two resonances clearly visible on each tuning curve. The higher frequency peak is from the pure optical resonance discussed here. The frequency of this pure optical resonance is set by microscopic tuning of the signal recycling mir-

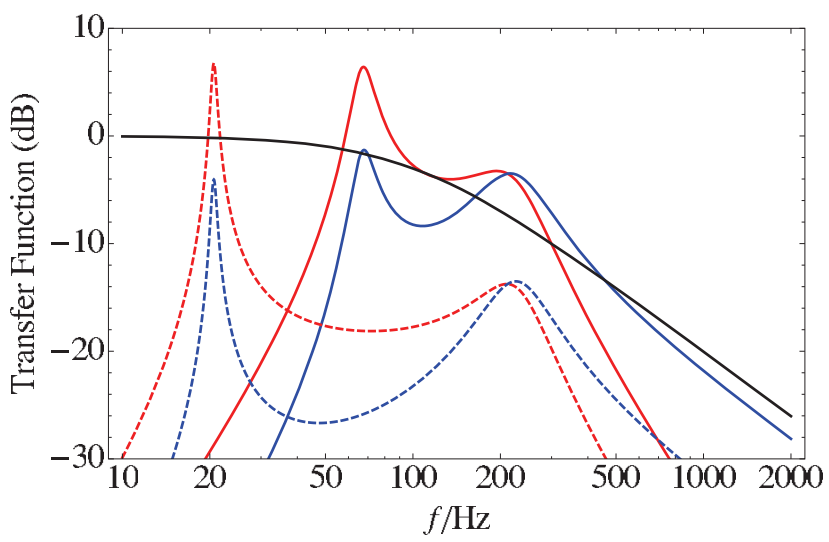

Figure 5 (online color at: www.lpr-journal.org) Response curves for a dual recycled Michelson interferometer showing optical (higher frequency) and optomechanical (lower frequency) resonances for 2 different laser powers. Solid curves are plotted with 10 times input power used in the broken curves. The two traces at each power level correspond to different measurement quadratures. The black curve with no peaks is the response at the higher power level without signal recycling. ror position. Enhancement at this resonance is determined by the finesse of the signal recycling cavity, mainly set by the reflectivity of the signal recycling mirror. Away from the peak, response rolls off with signal cavity bandwidth. The peak at lower frequency results from a radiation pressure driven opto-mechanical interaction. This effect was ignored in early analyses of signal recycling and resonant sideband extraction (see below). It will be discussed in detail in Sect. 5. Signal recycling is currently implemented on GEO600 [31].

Refinements of signal recycling include replacing the SM with an optical element whose reflectivity is variable [32], such as a short cavity or Michelson interferometer. This allows for tailoring of both the peak response frequency and of the interferometer bandwidth. By tuning an output cavity to the carrier frequency and making this element long enough so that the storage time of the signal can approach that of the storage time in the signal recycling cavity, the coupled cavity response enables resonant enhancement of both the upper and the lower GW sidebands.

In a major conceptual leap, Mizuno et al in 1993 [33] realized that signal recycling could be used to broaden the response of an interferometer. We have seen above that signal response is proportional to the stored power in the arm cavities. Ideally then we would like the cavity finesse to be as high as possible. The problem is that if the signal sidebands are stored too long in an arm cavity, GW induced phase change will average out. In an arm cavity Michelson interferometer with SR, the carrier field build up (storage) in the arm cavity is determined only by the reflectivity of the inboard arm cavity mirror. However, for the signal sidebands this reflectivity can be varied by changing the resonance condition of the signal in the SRC and hence the signal storage time can be altered. We are thus free to choose the in board mirror reflectivity as high as possible to build up carrier power, then tune the SRC to achieve the required signal bandwidth. This is termed Resonant Sideband Extraction (RSE).

The optical configuration chosen for second generation detectors now under construction (Advanced LIGO and Advanced Virgo) is that of an arm cavity Michelson interferometer with power- and signal-recycling. Third generation detectors are likely to use variants of these techniques that include modifications that allow for quantum non-demolition readout schemes.

\section{Quantum states of light and shot noise}

In a quantum statistical picture of light, the distribution of photons from a perfect laser (no classical amplitude or phase noise) obeys Poisson statistics in which the uncertainty in an ensemble of $n$ photons is given by the $\sqrt{n}$. This error in photon counting limits how small a mirror motion induced change in the number of photons measured at the output of a Michelson interferometer can be determined and is often referred to as shot noise or imprecision.

A more useful picture for understanding the role of quantum noise in a gravitational wave detector comes from 
quantizing the electromagnetic field into energy levels given by $E=(n+0.5) \hbar \omega$ and realizing that in the absence of photons $(n=0)$ there is still $0.5 \hbar \omega$ of energy in the electromagnetic vacuum modes. States of light are described by the creation and annihilation operators $\hat{a}^{\dagger}$ and $\hat{a}$ from which quadrature operators $\hat{X}_{1}$ and $\hat{X}_{2}$ corresponding to the real and imaginary parts of the electric field are defined [34]:

$$
\hat{X}_{1}=\frac{\left(a+a^{\dagger}\right)}{2} ; \quad \hat{X}_{2}=\frac{-i\left(a^{\dagger}-a\right)}{2} .
$$

The amplitude and phase quadratures represent non-commuting observable parameters. An operator for an arbitrary quadrature, $\zeta$, can be defined using a linear combination of $\hat{X}_{1}$ and $\hat{X}_{2}$

$$
\hat{X}_{\zeta}=\hat{X}_{1} \cos \zeta+\hat{X}_{2} \sin \zeta .
$$

Eq. (5) links the amplitude quadrature $\hat{X}_{1}$ and the phase quadrature $\hat{X}_{2}$ to the Hamiltonian of the harmonic oscillator, also providing a normalization in such a way that the quadrature variances for a vacuum state directly provide the zero point energy [35]

$$
\hat{H}=\hbar \omega(\hat{n}+1 / 2)=\hbar \omega\left(\hat{X}_{1}^{2}+\hat{X}_{2}^{2}\right),
$$

where $\hbar$ is Planck's constant, $\omega$ the oscillator's angular frequency and $\hat{n}$ the photon number operator. Eq. (6) displays the Heisenberg Uncertainty relation for the quadrature operator variances

$$
\left(\Delta \hat{X}_{1}\right)^{2} \cdot\left(\Delta \hat{X}_{2}\right)^{2} \geq \frac{1}{16}
$$

For a coherent state that exhibits Poisson statistics, the variance is the same in each quadrature giving

$$
\Delta \hat{X}_{1}=\Delta \hat{X}_{2}=\frac{1}{4} \text {. }
$$

On a phasor diagram, a classical laser beam is represented by a phasor with a precise length representing the amplitude and a well defined phase. In the quantum world, Eq. (7) requires that the phasor carry a Gaussian distributed noise at its end, representing a quantum uncertainty in its quadrature values, as depicted in Fig. 6. Even in the absence of the coherent "stick" i. e. a vacuum state, the "circle" of quantum noise remains. In the frequency domain (Fig. 7),

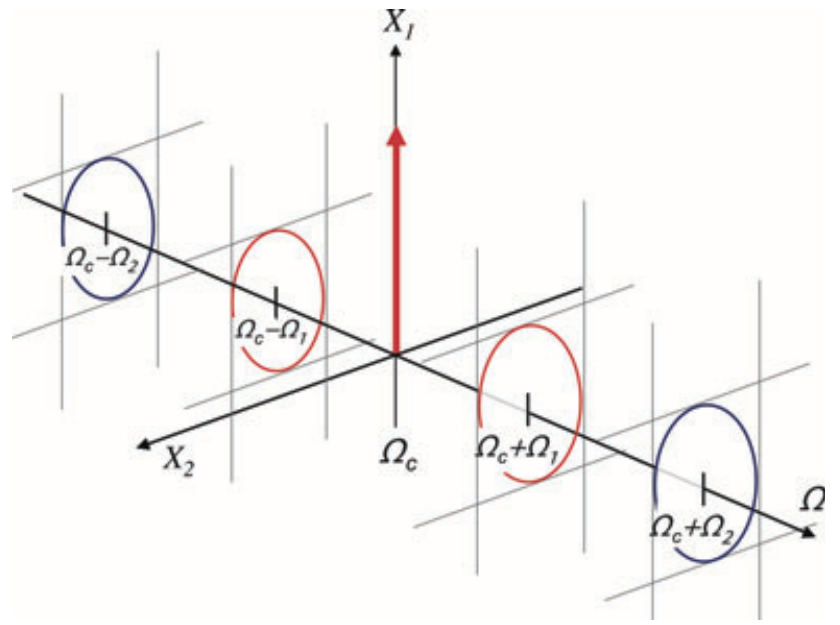

Figure 7 (online color at: www.lpr-journal.org) Two mode frequency domain representation of quantum noise for a (displaced) coherent state at optical frequency $\Omega_{c}$. The quantum noise of the carrier $(c)$ at a Fourier frequency $\Omega_{i}$ is given as the beat between upper and lower sidebands at optical frequencies $\Omega_{c} \pm \Omega_{i}$, all being in a vacuum state. Note, that the two sideband pairs shown here are just examples of a continuous spectrum of Fourier frequencies.

quantum noise is manifested through the beating of the coherent amplitude at carrier (zero) frequency, $\Omega_{c}$, with the upper and lower quantum noise sidebands in vacuum states at frequencies $\pm \Omega_{i}$ [34].

A quantum state is called a "squeezed state" [34] if $\Delta \hat{X}_{\zeta}<1$ for an arbitrary field quadrature $\hat{X}_{\zeta}$. Here, $\zeta$ is the squeezing angle. The greatest factor by which the variance is below 1 is called the squeezing factor, often given on a decibel scale. Squeezed states belong to the class of so-called nonclassical states $[34,35]$. In such states the detection events of photons are not independent from each other, but show quantum correlations. Figure 8 displays the noise distribution for an amplitude squeezed state with the same coherent displacement. In the frequency domain, the quantum noise of the upper and lower sideband at Fourier frequency $\Omega$ is still random but mutually correlated within a sideband pair. For an amplitude squeezed state, the sidebands are amplitude modulation anti-correlated and phase modulation

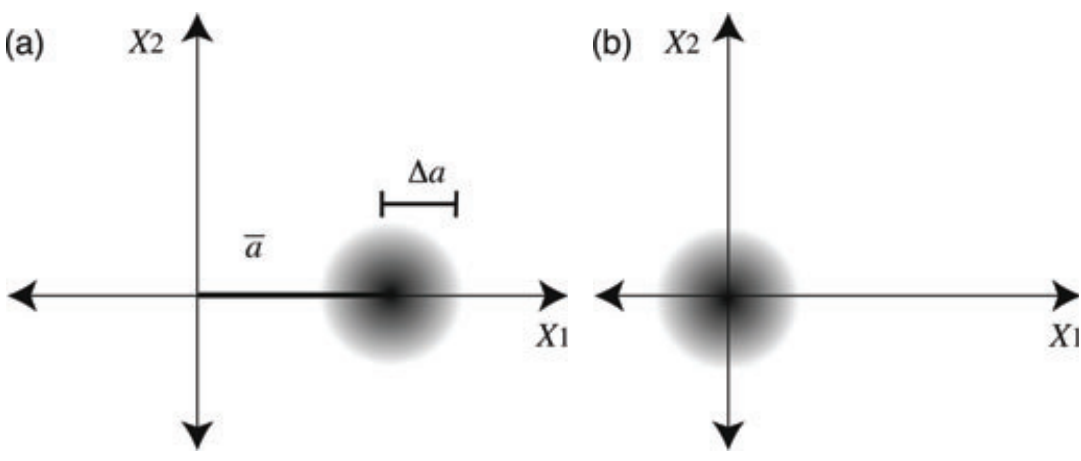

Figure 6 Single mode phasor representation showing (a) quantum noise for a coherent state and (b) vacuum state.

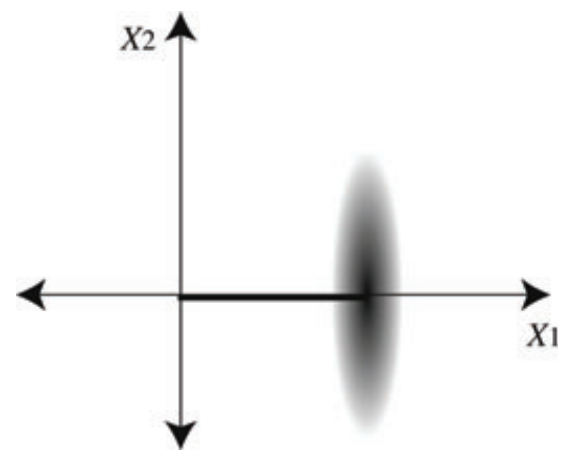

Figure 8 Phasor representation showing displaced amplitude squeezed quantum noise. 


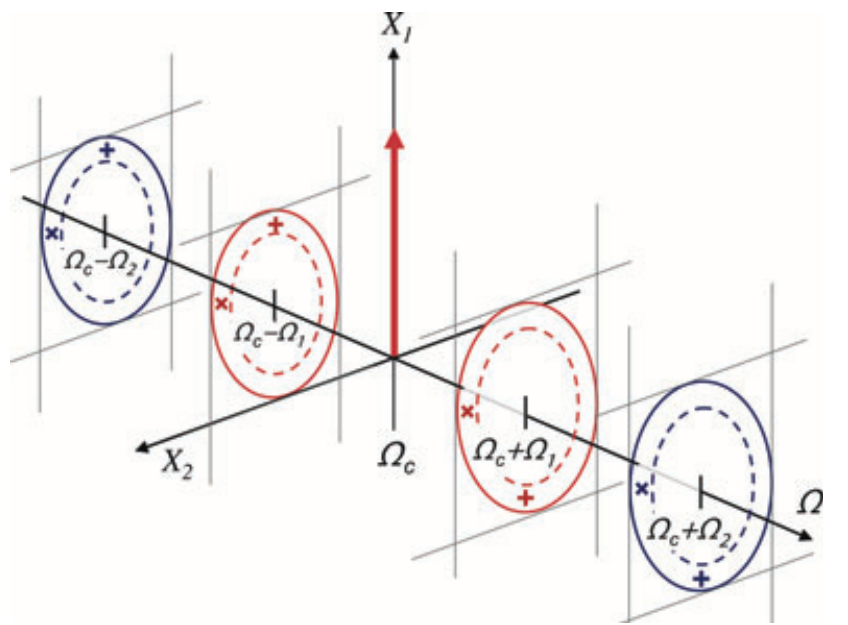

Figure 9 (online color at: www.lpr-journal.org) Frequency domain representation of quantum noise for a displaced amplitude squeezed state at optical frequency $\Omega_{c}$. Squeezing is produced by optical parametric amplification (parametric down conversion) and increases the quantum noise at all frequencies $\Omega_{c} \pm \Omega_{i}$. This noise is quantum correlated such that every sideband pair results in a squeezed noise at a single-sided Fourier frequency $\Omega_{i}$. Note, that quantum correlations in the imaginary direction are displayed by the (correlated) position of the symbol $\times$ within the uncertainty circle, and quantum anti-correlations in the real valued direction are displayed by the (anti-correlated) position of the symbol + .

correlated, as depicted in Fig. 9. Squeezing increases the quantum noise at all frequencies $\Omega_{c} \pm \Omega_{i}$. However, this noise is quantum correlated such that every sideband pair results in a squeezed noise at Fourier frequency $\Omega_{i}$.

Using the phasor picture we see that the difference in phase between two phasors, i. e. the signal from an interferometer, can only be resolved to the width of the circle of quantum noise each carries. For a coherent state, this is referred to as the shot noise limit. In the early 1980s Caves $[36,37]$ went further in his analysis of a Michelson interferometer to show that, when operating on a dark fringe, the circle of quantum noise in fact comes from the vacuum field which enters the interferometer through the open output port. All noise entering with the pump laser (classical and quantum) is common to both arms of the Michelson interferometer and, therefore, does not transmit to the output (dark) port (assuming perfect fringe contrast).

Interpreting quantum noise as coming from vacuum fluctuations means that the size of the noise does not change with laser power. As a result, it is clear that increasing the laser power makes the coherent amplitudes larger and hence the minimum resolvable phase difference between two phasors becomes smaller. Increasing the laser power reduces the influence of shot noise. A full analysis gives the shot noise power spectral density for a simple Michelson interterometer, $S_{\mathscr{Z} \mathscr{Z}}^{\mathrm{MI}}$, as [38]:

$$
S_{\mathscr{Z} \mathscr{Z}}^{\mathrm{MI}}=\frac{\lambda}{16 \pi} \frac{\hbar c}{I_{0}}
$$

We see that shot noise limited sensitivity (square root of the power spectral density) scales with the inverse of the square root of the laser power $I_{0}$ and is independent of frequency, i. e. it is white.

In fact, at the signal output port of a laser interferometer, photo-electric detection acquires information about just one quadrature of the output field. This quadrature is the amplitude quadrature corresponding to the light's phase quadrature in the interferometer arms. Thus by replacing the vacuum state with phase squeezed vacuum with respect to the output's amplitude quadrature, the light fields in the interferometer become entangled [39], a more precise measurement of optical phase difference can be made, surpassing the shot noise limit. Accordingly, the noise in the orthogonal field quadrature is increased.

\section{Radiation pressure noise, the SQL, and beyond}

The laser power cannot be increased without limit, however. Quantum back action, the impact of the measurement on the device, in the form of quantum radiation pressure noise intervenes $[6,36]$. In an interferometer with suspended mirrors, photon pressure can move the mirrors. Radiation pressure from the driving field is common in the Michelson interferometer arms and does not generate a (differential) interferometer signal. The relevant radiation pressure force arises from the beat of the coherent drive with amplitude vacuum fluctuations entering through the dark port. This fluctuating force randomly differentially buffets the mirrors. The resulting noise increases with the square root of the laser power. Since it is anticorrelated in each arm, its effect does not cancel at the beam splitter. At some power level, the random motion induced by quantum radiation pressure noise will dominate shot noise masking the GW-induced motion.

Measurement-induced back action starts to become important at a scale determined by the quantum mechanics of the test mass. Fundamentally, this is because light couples to the position $x$ of a nearly free mass, whose Heisenberg operators (denoted by the subscript $H$ ) at different times do not commute:

$$
\left[\hat{x}_{H}(t), \hat{x}_{H}\left(t^{\prime}\right)\right]=\frac{i \hbar\left(t^{\prime}-t\right)}{M} .
$$

According to the fundamental postulate of quantum mechanics regarding measurement, this means the observables $x(t)$ and $x\left(t^{\prime}\right)$ cannot both be determined precisely without any additional noise. Mathematically, this leads to

$$
\Delta x\left(t_{1}\right) \cdot \Delta x\left(t_{2}\right) \geq \frac{\hbar\left|t_{2}-t_{1}\right|}{2 M} .
$$

This non-commutativity then leads to the Standard Quantum Limit. Suppose we measure the change in position between two instants separated by $\tau$, in order to measure a gravitational-wave pulse during this time, we then have

$$
x_{\mathrm{GW}} \sim x\left(t_{2}\right)-x\left(t_{1}\right)
$$


and

$$
\begin{aligned}
\Delta x_{\mathrm{GW}} \sim & \sqrt{\Delta x^{2}\left(t_{2}\right)+\Delta x^{2}\left(t_{1}\right)} \\
& \geq \sqrt{2 \Delta x\left(t_{2}\right) \cdot \Delta x\left(t_{1}\right)} \geq \sqrt{\frac{\hbar \tau}{M}} \equiv \Delta x_{\mathrm{SQL}} .
\end{aligned}
$$

A rigorous derivation gives the power spectral density of the SQL as a function of angular frequency $\Omega$ for a simple Michelson interferometer as:

$$
S_{x}^{\mathrm{SQL}}=\frac{2 \hbar}{M \Omega^{2}} .
$$

In an actual measurement process, non-simultaneous measurability of $x(t)$ and $x\left(t^{\prime}\right)$ is enforced by additional noise arising from quantum fluctuations of the optical field. In fact, Eq. (9) implies that, when coupled with an external operator, the evolution of $x$ must be "contaminated" by fluctuations from the optical system:

$$
\hat{x}_{H}^{(1)}(t)=\hat{x}_{H}^{(0)}(t)+\frac{i}{\hbar} \int_{0}^{t} d t^{\prime}\left[\hat{x}_{H}^{(0)}(t), \hat{x}_{H}^{(0)}\left(t^{\prime}\right)\right] \hat{F}^{(1)}\left(t^{\prime}\right) .
$$

Here $\hat{x}^{(1)}$ is the Heisenberg operator of mirror position when it couples to the optical system, $\hat{x}^{(0)}$ is the "free evolution" without coupling to measurement device, and $\hat{F}^{(1)}$ is the radiation-pressure force acting on the mirror. Equation (14) indicates the existence of the back-action noise, which arises when the measurement system exerts a force back onto the system being measured - in our case the fluctuating part of $\hat{F}^{(1)}$.

On the other hand, if the optical observable $F$ has

$$
\left[\hat{F}_{H}^{(0)}(t), \hat{F}_{H}^{(0)}\left(t^{\prime}\right)\right] \neq 0
$$

the test mass also influences the evolution of $F$, with

$$
\hat{F}_{H}^{(1)}(t)=\hat{F}_{H}^{(0)}(t)+\frac{i}{\hbar} \int_{0}^{t} d t^{\prime}\left[\hat{F}_{H}^{(0)}(t), \hat{F}_{H}^{(0)}\left(t^{\prime}\right)\right] \hat{x}^{(1)}\left(t^{\prime}\right) .
$$

Equation (16), together with Eq. (14), shows that not only does the measurement system injects noise - it also modifies the dynamics of the system being measured, through the coupled evolution of the non-fluctuating parts of $\hat{x}^{(1)}$ and $\hat{F}^{(1)}$. In the next two sections, we will address backaction noise and modification of dynamics, respectively.

\subsection{General discussion of the SQL}

As stated earlier, in laser interferometers, back action noise takes the form of radiation pressure noise, which arises from photons randomly hitting the mirrors and creating noisy motion. The full measurement result, assuming homodyne detection [34] of an out-going field quadrature, can be written in the frequency domain as,

$$
y(\Omega)=x(\Omega)+\mathscr{Z}(\Omega)+R_{x x}(\Omega) \mathscr{F}(\Omega) .
$$

Here $x$ is the position of the mirror were there no measurement, which in turn contains free motion of the mirror plus gravitational-wave-induced motion; $\mathscr{Z}$ is shot noise, and $\mathscr{F}$ is radiation-pressure force. The quantity

$$
R_{x x}=-\frac{1}{M \Omega^{2}}
$$

is the response of a free mass to external force. The operators $\mathscr{Z}$ and $\mathscr{F}$ have the following commutation relations:

$$
\begin{aligned}
& {\left[\mathscr{Z}(t), \mathscr{Z}\left(t^{\prime}\right)\right]=\left[\mathscr{F}(t), \mathscr{F}\left(t^{\prime}\right)\right],} \\
& {\left[\mathscr{Z}(t), \mathscr{F}\left(t^{\prime}\right)\right]=i \delta\left(t-t^{\prime}\right) .}
\end{aligned}
$$

These relations guarantee that the full measurement result $y$ commutes at different times:

$$
\left[y(t), y\left(t^{\prime}\right)\right]=0 .
$$

The commutation relation (19) also gives rise to a Heisenberg Uncertainty relation among the spectra of $\mathscr{Z}$ and $\mathscr{F}$ :

$$
S_{\mathscr{Z} \mathscr{Z}} S_{\mathscr{F} \mathscr{F}}-\left|S_{\mathscr{Z} \mathscr{F}}\right|^{2} \geq \hbar^{2} .
$$

Upon assuming uncorrelated sensing and back-action noise, or $S_{\mathscr{Z} \mathscr{F}}=0$, we get

$$
S_{\mathscr{Z} \mathscr{Z}} S_{\mathscr{F} \mathscr{F}} \geq \hbar^{2},
$$

and in this case,

$$
S_{y}=S_{\mathscr{Z} \mathscr{Z}}+\left|R_{x x}\right|^{2} S_{\mathscr{F} \mathscr{F}} \geq 2 \hbar\left|R_{x x}\right| \equiv S_{x}^{\mathrm{SQL}} .
$$

So far, we have seen that $\left[x(t), x\left(t^{\prime}\right)\right] \neq 0$ is connected with $R_{x x} \neq 0$, which in turn gives the SQL. As a consequence, one obvious way to defeat the SQL is to measure a quantity $A$ of the mirror that does commute at different times, i. e.

$$
\left[A(t), A\left(t^{\prime}\right)\right]=0
$$

where $A$ could be the momentum of the mirrors. Such a quantity $A$ is called a Quantum Non-Demolition (QND) observable. However, for a long time, it was not clear how we can measure momentum of free masses in large-scale laser interferometers. In Sect. 4.4, we will introduce speed meter interferometers, which can be viewed as measuring momentum, but only do so in an effective way. On the other hand, the fundamental coupling of light to test masses has always been through position, and, therefore, all configurations that surpass the SQL can all be viewed as circumventing the SQL by introducing correlations between the shot noise $\mathscr{Z}$ and radiation-pressure noise $\mathscr{F}$. We note that injecting squeezed light can also result in beating the SQL [40-42]. In this case the correlation is introduced by correlating the amplitude and phase quadratures prior to insertion into the interferometer. Nevertheless, for historical reasons, these interferometers are sometimes still referred to as QND interferometers. 


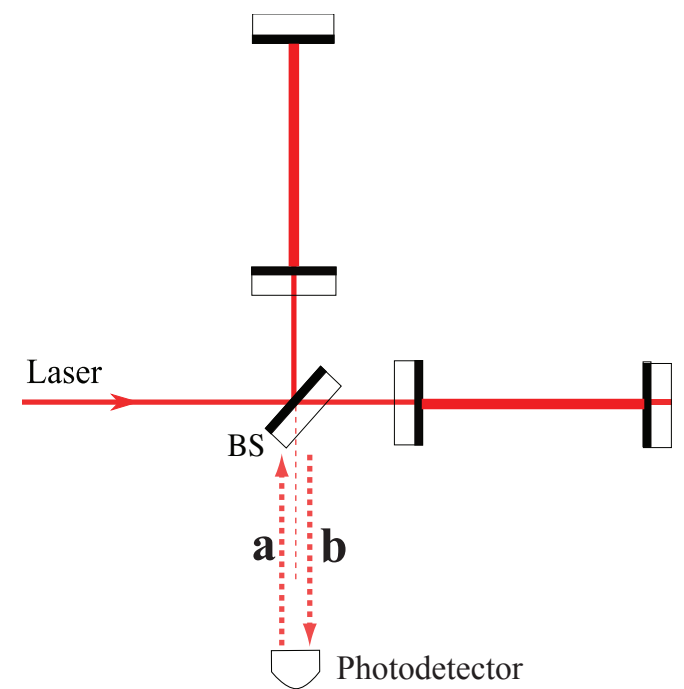

Figure 10 (online color at: www.lpr-journal.org) Schematic diagram of a Fabry-Perot Michelson interferometer showing the input field quadratures, $\mathbf{a}=\left(a_{1}, a_{2}\right)$, into the detection port and the output field quadratures, $\mathbf{b}=\left(b_{1}, b_{2}\right)$, from the detection port.

\subsection{Fabry-Perot Michelson interferometers}

For a more concrete example, let us consider a Fabry-Perot MI, as shown in Fig. 10. Upon writing electric field as quadratures:

$$
E(t)=\sqrt{\frac{4 \pi \hbar \Omega_{c}}{\mathscr{A} c}}\left[a_{1}(t) \cos \Omega_{c} t+a_{2}(t) \sin \Omega_{c} t\right]
$$

where $\mathscr{A} c$ is the cross-sectional area of the beam of frequency $\Omega_{c}$, we can write down the input-output relation, i. e., how the outgoing field quadratures $\left(b_{1}, b_{2}\right)$ relate to the incoming quadratures $\left(a_{1}, a_{2}\right)$ at the detection port in the presence of a gravitational-wave of amplitude $h$ [43]:

$$
\begin{aligned}
& b_{1}=e^{2 i \beta} a_{1} \\
& b_{2}=e^{2 i \beta}\left(a_{2}-\mathscr{K} a_{1}\right)+e^{i \beta} \sqrt{2 \mathscr{K}} \frac{h}{h_{\mathrm{SQL}}} .
\end{aligned}
$$

Here $\beta=\arctan \Omega / \gamma$, where $\gamma$ is the bandwidth of the arm cavity,

$$
\mathscr{K}=\frac{2 \gamma \Theta^{3}}{\Omega^{2}\left(\Omega^{2}+\gamma^{2}\right)}, \quad \Theta^{3}=\frac{8 \omega_{0} I_{0} / \mathscr{T}_{i}}{m L c},
$$

and

$$
h_{\mathrm{SQL}}=\sqrt{\frac{8 \hbar}{m \Omega^{2} L^{2}}}
$$

where $I_{0}$ is input power of the laser and $\mathscr{T}_{i}$ is the power transmission coefficient of the cavity input mirror. The dimensionless quantity $\mathscr{K}$, proportional to the optical power characterizes the measurement strength.

In Eqs. (26) and (27), the phase factor $e^{2 i \beta}$ arises from the arm-cavity-induced time delay between incoming and outgoing fields. We see that the phase quadrature of $b$ contains the signal and depends on both the phase and the amplitude quadratures of the field entering the antisymmetric port, $a$. In Eq. (27) for the phase quadrature: the term proportional to $h$ arises from the mirror's GW-induced motion phase modulating the carrier; the term proportional to $a_{2}$ gives rise to shot noise; the term proportional to $\mathscr{K}$ in Eq. (27) is back-action noise.

In the case of the Fabry-Perot MI, for an input vacuum state, the quadratures of the input field, $\left(a_{1}, a_{2}\right)$, are uncorrelated. We have $S_{a_{i} a_{j}}=\delta_{i j}$, and we detect output phase quadrature $b_{2}$ (the quadrature that contains signal), we have an $h$-referred noise spectrum of

$$
S_{h}=\left[\frac{1}{\mathscr{K}}+\mathscr{K}\right] \frac{h_{\mathrm{SQL}}^{2}}{2}
$$

where the first term, inversely proportional to power, is shot noise related, while the second term, directly proportional to power, is radiation-pressure noise related. Together, the total noise is limited by the SQL. One way to surpass the SQL is to inject a squeezed vacuum state into the dark port of the interferometer (input squeezing), for which the $a_{2}-\mathscr{K} a_{1}$ quadrature is squeezed. Because $\mathscr{K}$ is highly frequency dependent, this quadrature will also be frequency dependent. Qualitatively, at high frequencies, $\mathscr{K} \ll 1$, shot noise dominates, and we need to squeeze $a_{2}$, while at low frequencies, radiation-pressure noise dominates, $\mathscr{K} \gg 1$, and we need to squeeze $a_{1}$. Another way is to detect an appropriate combination between $b_{1}$ and $b_{2}$,

$$
b_{\zeta}=b_{1} \cos \zeta+b_{2} \sin \zeta
$$

in such a way that the $a_{1}$ content of $b_{1}$ (which is shot noise) cancels with the $a_{1}$ content of $b_{2}$ (which is back-action noise). Any particular $\zeta$ can be implemented by using a local oscillator with phase $\zeta$. Here the optimal detection quadrature also moves from $b_{2}$ to almost $b_{1}$, when frequency decreases. The optimal $\zeta$ is frequency dependent. Aside from the matter of frequency dependence, both approaches successfully circumvent the SQL by introducing correlation between radiation-pressure and shot noise in the outgoing field.

\subsection{Optical filters}

In order to achieve the frequency dependence of the above approaches to SQL-beating, Kimble et al. [43] proposed using detuned Fabry-Perot cavities as optical filters, which allow frequency-dependent rotations of quadratures by a substantial amount when the side-band frequency crosses resonance. Known as variational readout, Purdue and Chen [44] worked out the general frequency dependence realizable by a series of such filters. In the case of Fabry-Perot Michelson interferometers, in general two filter cavities are required (Fig. 11), with detuning and bandwidth in the detection band of the interferometer (around $100 \mathrm{~Hz}$ ). Figure 12 compares the sensitivity achievable without and with filter cavities. 


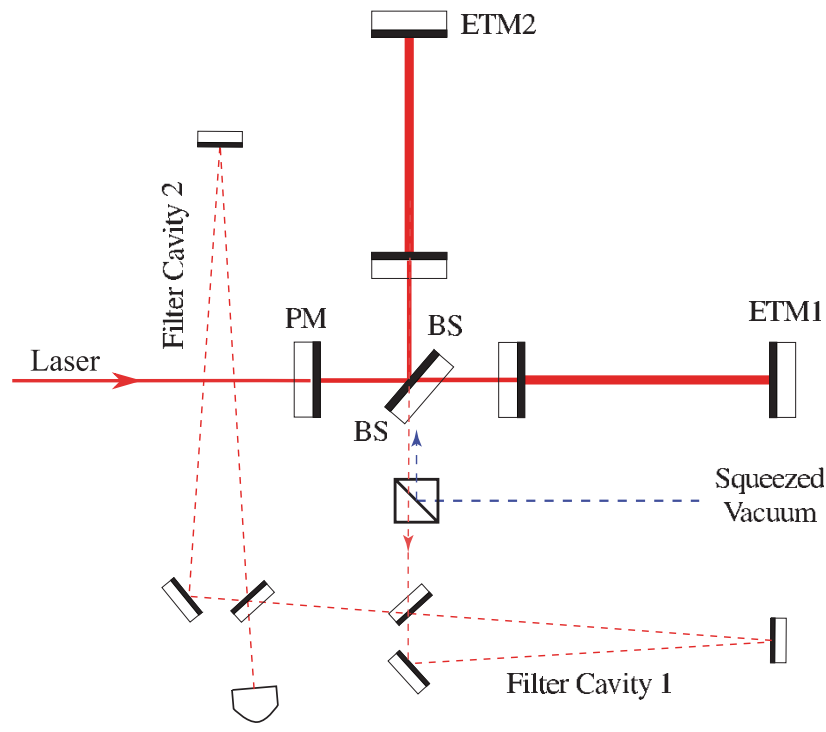

Photodetector

Figure 11 (online color at: www.lpr-journal.org) Schematic of the layout of a power recycled Michelson interferometer with filter cavities inserted between the output port and the Homodyne detector. The filter cavities will both rotate the signal and the injected squeezed noise into the optimum measurement quadrature

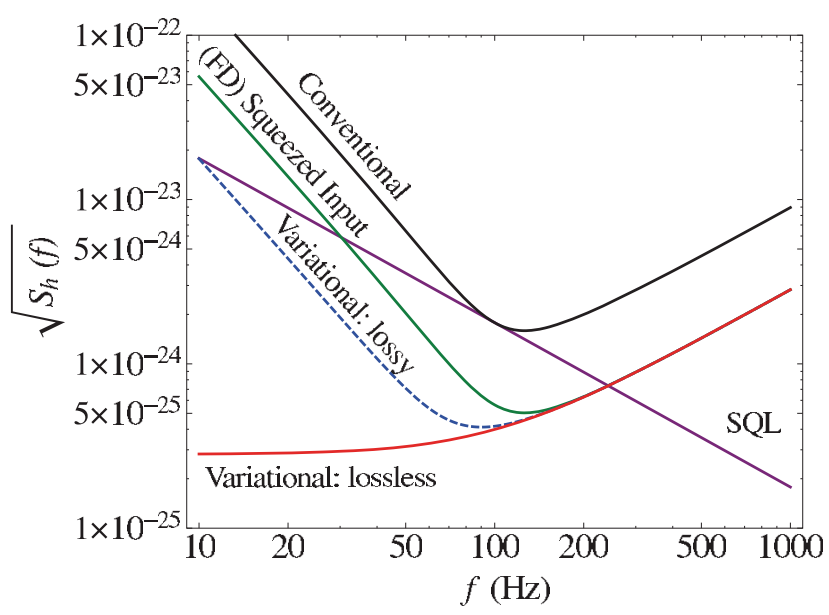

Figure 12 (online color at: www.lpr-journal.org) Sensitivity enhancement of a power recycled Michelson interferometer without filter cavities (black trace), with $10 \mathrm{~dB}$ squeezed light and filter cavities (solid green trace); with $10 \mathrm{~dB}$ squeezing and variational readout (solid red trace) and finally, $10 \mathrm{~dB}$ squeezing and variational readout but including small losses in the filter cavities (broken blue trace).

A broadband nonclassical noise reduction beyond the SQL can be achieved if the injection of squeezed light is combined with additional narrowband optical filter cavities at the interferometer dark port $[43,45]$. In order to achieve such narrow bandwidth with a rather low optical loss (crucial for maintaining quantum coherence in the squeezed vacuum and the outgoing quadratures), these filter cavities will have to be kilometers in scale. Even so, as we see in Fig. 12, these interferometers are still significantly limited by optical losses in the interferometer. As a rule of thumb, the factor by which an interferometer can surpass the SQL through back-action evasion is

$$
\sqrt{S_{h}} \geq\left(e^{-2 r} \varepsilon\right)^{1 / 4} h_{\mathrm{SQL}}
$$

where $e^{-2 r}$ is the power squeeze factor, and $\varepsilon$ the total loss of the interferometer, in power.

Due to the high cost of constructing km-scale cavities, sub-optimal filtering schemes have been considered. For example, Corbitt, Mavalvala and Whitcomb [46] considered amplitude filters that only inject phase-squeezed vacuum at high sideband frequencies, and amplitude-squeezed vacuum at low sideband frequencies - using impedance-matched cavities. This scheme is less susceptible to losses, intuitively because it simply needs to direct the input squeezing around, instead of having to rotate it with delicate phases. The amplitude filter scheme initially leaves one port where light exits freely; Khalili [47] proposed to insert an additional homodyne detection here as an auxiliary channel, further improving this scheme.

\subsection{Speed meter interferometers}

Momentum of a free mass is a so-called QND observable, and, therefore, could be measured without imposing backaction. In the 1990s, Braginsky and Khalili [48] proposed configurations in which speed of a free mass could be measured, yet these were not clearly implementable in largescale laser interferometers. In the first scheme, two resonators with initially the same eigenfrequency are weakly coupled to each other, forming a system with two closely split eigenfrequencies. When oscillator A is driven directly, its response will slowly slosh into B, and slosh back, with a 180-degree phase shift compared with the directly driven signal. Such a sloshing makes the two-resonator system only sensitive to position change (and hence speed) induced by the driving force. An appropriate choice of sloshing time scale and signal extraction time scale will give rise to a speed meter at frequencies below the sloshing frequency. We shall denote this the "two-resonator" scheme. In a second scheme, opposite sides of the same test mass are sensed with a time delay, therefore giving rise to speed measurement. We shall denote this the "two-bounce" scheme.

Purdue [49] made the first attempt to convert the tworesonator scheme into kilometer-scale interferometers, and Purdue and Chen [44] designed the first practical version of a speed meter, which consisted of an additional cavity at the dark port of a MI. Chen then showed that the two bounce scheme could naturally be realized by a Sagnac interferometer with arm cavities and signal recycling. The layout is shown in Fig. 13.

In essence, after the beam splitter the horizontal field senses ETM1 at time $t$, and ETM2 at some time later $t+\tau$. On the other hand, the vertical field first senses ETM2 at time, $t$, and ETM1 at some later time $t+\tau$. As the mirror positions are sensed at different times, the resulting phase difference is effectively a measure of speed. 


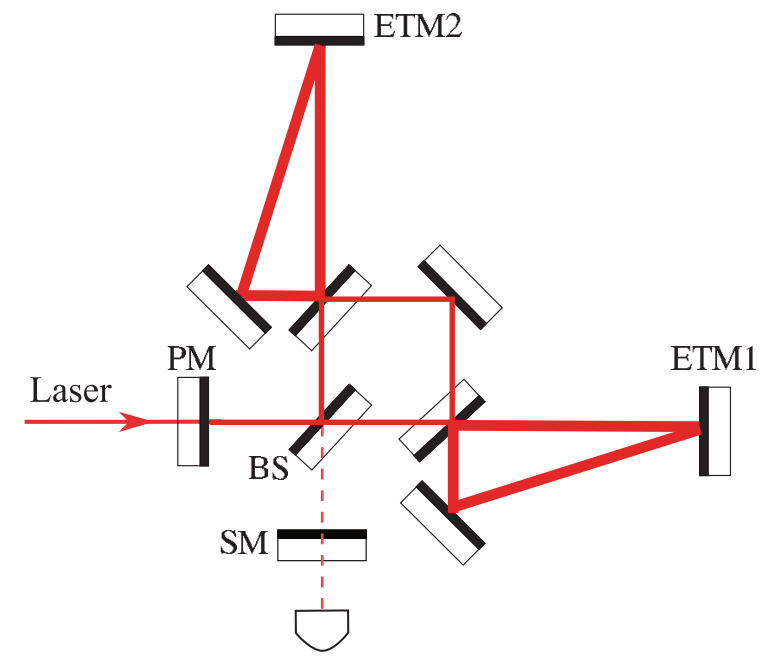

Photodetector

Figure 13 (online color at: www.lpr-journal.org) Optical schematic for a Sagnac speed meter comprising a Sagnac interferometer with ring cavities at two vertices.

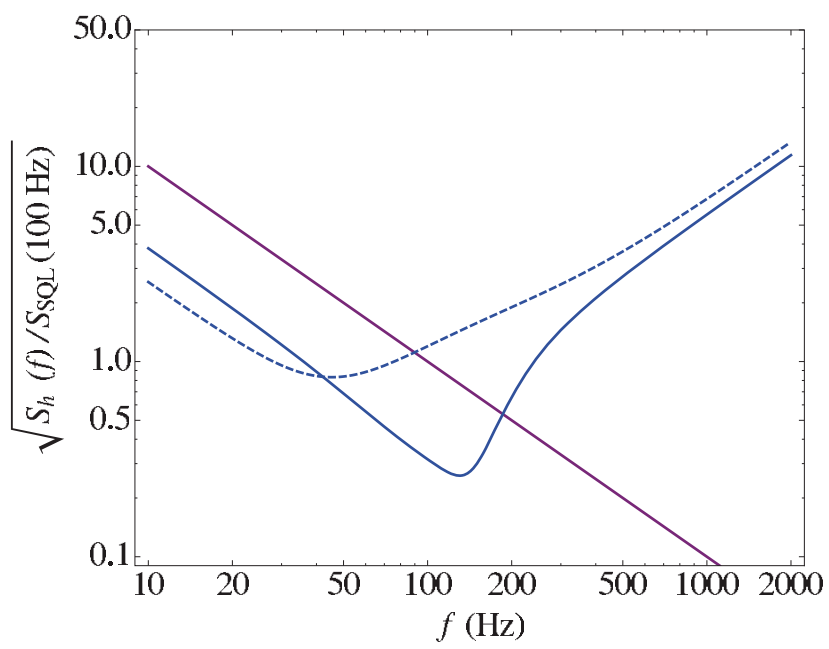

Figure 14 (online color at: www.lpr-journal.org) Sagnac speed meter sensitivity. Straight line: locus of the SQL for a range of optical power; broken curve: the Sagnac speed meter beats the SQL below $100 \mathrm{~Hz}$; solid curve: Sagnac speed meter with signal recycling beats the SQL below $200 \mathrm{~Hz}$. Reproduced from [50], Fig. 6 .

Chen [50] further showed that the resulting interferometers have mathematically equivalent input-output relations, both very similar to Eqs. (26) and (27), but with $\mathscr{K}$ approximately a constant value for a broad frequency band, instead of rising monotonically in the case of Fabry-Perot Michelson interferometers. More specifically, for a speed meter, we have

$$
\mathscr{K}=\frac{8 \delta \Theta^{3}}{\left(\Omega^{2}-\Omega_{*}^{2}\right)^{2}+\Omega^{2} \delta^{2}}
$$

where $\Omega_{*}$ is the sloshing frequency, and $\delta$ the signal extraction rate.
As is easily seen from the frequency-domain formula (27), a constant $\mathscr{K}$ in a broad frequency band corresponds to speed measurement over a broad frequency and (because $h_{\mathrm{SQL}} \sim 1 / \Omega$ ). Interestingly, just from Eq. (27), the speed meter is not free from radiation-pressure noise: the mirror still moves randomly, driven by radiation-pressure force. However, in the region where $\mathscr{K}$ is constant, radiationpressure noise is easily canceled with shot noise, if we detect the appropriate constant quadrature. This need for canceling among radiation-pressure and shot noise can be explained by the fact that whenever we couple to speed of a free mass, its canonical momentum (which is conserved) becomes different from its kinetic momentum by a term involving the light field - and kinetic momentum is no longer conserved. The quadrature which contains no back action actually senses the canonical momentum, and in this way the speed meter can be viewed as a QND device.

\section{Radiation pressure modification of mirror dynamics}

So far, we have examined the interaction of the optomechanical system with quantum noise. However, in the presence of signal, it is possible for the mechanical system to be resonantly excited, thereby increasing sensitivity. Classical radiation pressures forces play a central role in these phenomena, and are discussed below.

\subsection{Classical optical forces}

If an optical cavity is exactly on resonance, the intracavity power depends quadratically on small changes in the length of the cavity, and the restoring force is only a secondorder effect on the dynamics of the cavity (see Fig. 15). The constant (DC) radiation pressure is balanced through external forces; consequently, only fluctuations of the radiation pressure are considered. In an optical cavity that is detuned from resonance, the intracavity power, and, therefore, the radiation pressure exerted on the mirrors, becomes linearly dependent on the length of the cavity, analogous to a spring. The resulting "optical spring" has a spring constant in the frequency domain given by $[17,21]$

$$
\begin{aligned}
K(\Omega) & =K_{0} \frac{\left[1+(\delta / \gamma)^{2}-(\Omega / \gamma)^{2}\right]}{\left[1+(\delta / \gamma)^{2}-(\Omega / \gamma)^{2}\right]^{2}+4(\Omega / \gamma)^{2}} \\
K_{0} & =\frac{2}{c} \frac{d P}{d L}=\frac{128 \pi I_{0}(\delta / \gamma)}{\mathscr{T}_{i}^{2} c \lambda_{0}}\left[\frac{1}{1+(\delta / \gamma)^{2}}\right],
\end{aligned}
$$

where $\Omega$ is the frequency of the motion, $\lambda_{0}=1064 \mathrm{~nm}$ is the wavelength of light, $c$ is the speed of light, $\gamma$ is the cavity linewidth, $\mathscr{T}_{i}$ is the power transmission coefficient of the cavity input mirror, and $\delta$ and $I_{0}$ are the detuning and input power of the laser, respectively. Note the dependence of $K_{0}$ on the sign of $\delta$. For $\delta>0$ (in our convention), $K>0$ 

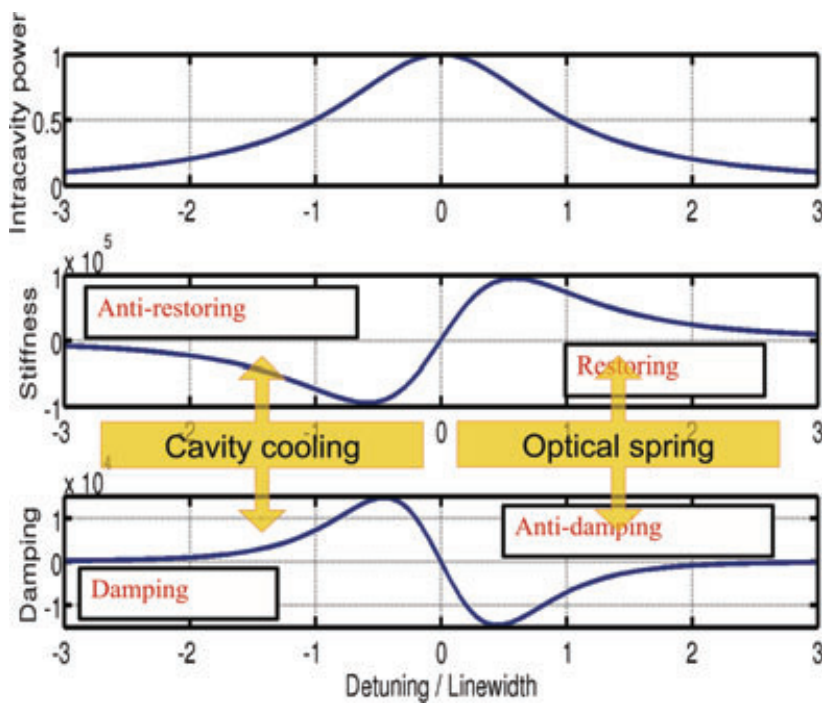

Figure 15 (online color at: www.lpr-journal.org) Intracavity power, and optical restoring and damping forces as a function of detuning of the laser from cavity resonance. Top panel: circulating power in the cavity; Middle panel: optical stiffness given by the real part of the total optical force; Bottom panel: optical damping given by the imaginary part of the total optical force. Red detuning (to below resonance) is the regime of cavity cooling, while blue-detuning (to above the resonance) gives rise to the optical spring.

corresponds to a restoring force, while $\delta<0$ gives an antirestoring force (see Fig. 15, middle panel).

The light in the cavity (for $\delta \ll \gamma$ ) responds to mirror motion on a time scale given by $\gamma^{-1}$. This delay has two effects. First, for high frequency motion $(\Omega \gtrsim \gamma)$, the response of the cavity, and the corresponding radiation pressure, are reduced, and we see from Eq. (34) that $K(\Omega \gg \gamma) \approx K_{0}(\Omega / \gamma)^{-2}$. Second, the response of the cavity lags the motion, leading to an additional force proportional to the velocity of the mirror motion - a viscous force with damping coefficient given by $[17,21]$

$$
\Gamma(\Omega) \equiv \frac{2 K(\Omega)}{M \gamma\left[1+(\delta / \gamma)^{2}-(\Omega / \gamma)^{2}\right]},
$$

where $M$ is the reduced mass of the two mirrors (see Fig. 15, bottom panel).

The optical spring effect leads to a modified resonant frequency and quality factor of the optomechanically coupled system, given by

$$
\begin{aligned}
& \Omega_{\text {eff }}^{2} \equiv \frac{K_{0} / M}{1+(\delta / \gamma)^{2}}, \\
& Q_{\text {eff }} \equiv\left(1+(\delta / \gamma)^{2}\right) \frac{-\gamma}{2 \Omega_{\text {eff }}} .
\end{aligned}
$$

Because the cavity response lags the motion of the mirrors, a restoring spring constant implies a negative damping. We see that when both optical forces dominate their mechanical counterparts, the system can become unstable (note that
$Q_{\text {eff }}$ is negative). The optical spring resonance can be stabilized either with electronic feedback [21], or with a second optical field that responds on different time scales [11].

\subsection{Opto-mechanics and signal recycling interferometers}

When a signal-recycling mirror is placed at the antisymmetric port of the Michelson Interferometer (see SM in Fig. 3), the differential optical mode may be detuned from resonance. This detuning, together with increased optical power (several hundred kilowatts in the arm cavity), makes the optical spring an important effect in such interferometers. Depending on the sign of detuning, the optical spring can move the $1 \mathrm{~Hz}$ mechanical resonance of the pendulum up to a few tens of Hertz. This is the origin of the low frequency peaks shown in Fig. 5 [16,51]. The power dependent stiffness of the optical spring is clearly evident in the shift of the opto-mechanical resonance to higher frequency when the laser power is increased. As mentioned in the previous section, this resonance is unstable due to anti-damping and must be controlled either via electronic or optical feedback. Alternatively, for the opposite sign of detuning, an instability will develop on a similar time scale.

Figure 16 shows sensitivity curves as the signal recycling cavity is detuned away from the carrier resonance (in the absence of all other noise sources). We see that the sensitivity is improved around both the optical spring resonance [16-25,51], and the pure optical resonance. Around the optomechanical enhancement, the free-mass Standard Quantum Limit is surpassed. Much of this improvement can be most straightforwardly attributed to resonant enhancement due to the spring constant.

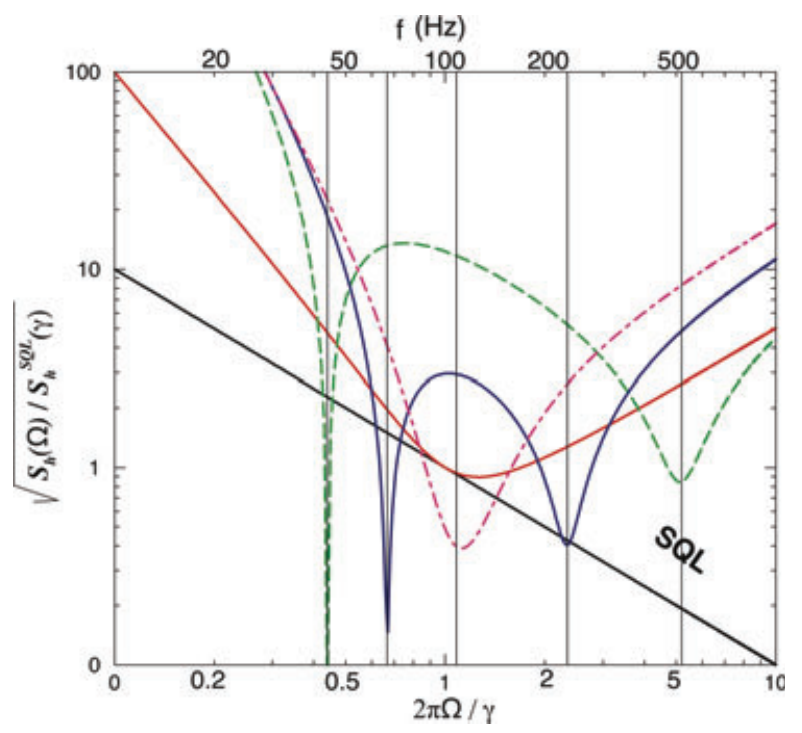

Figure 16 (online color at: www.lpr-journal.org) Optomechanical resonance enhancement with signal recycling. Black straight line is the SQL locus. Red curve: no signal recycling; Blue, green (dashed), and magenta (dash-dot) curves: varying detunings of the signal recycling cavity. 


\section{Quantum optics: Ponderomotive squeezing and optical entanglement}

Radiation-pressure-induced optical forces can be used to generate squeezed states of the electromagnetic field [19,20, 43]. A simple picture of squeezing due to optomechanical coupling can be understood as follows: amplitude fluctuations of the optical field impinging on the low-mass mirrors drive position fluctuations of the mirrors; as the light reflects of the mirrors, these position fluctuations are imposed on the phase of the optical field, thus correlating amplitude and phase fluctuations of the light. These amplitude-phase correlations lead to squeezing of the light. Mathematically, this effect is contained in Eqs. (26) and (27).

However, upon a more detailed consideration, from the frequency dependence of $\mathscr{K}$, it is evident that the squeezing factor and squeezing phase generated this way are highly frequency dependent, and not easily compatible with sensitivity enhancement in gravitational-wave detectors. One way of getting around this is to couple the mirror position degree of freedom to an optical spring, which makes the squeezing factor and squeezing angle constant below the optomechanical resonance $[19,20]$. The fluctuations in the cavity length due to external forces (such as seismic or thermal noise) are also greatly reduced due to the optical spring. It is this suppression factor, provided by the mechanically lossless optical spring, that makes it possible to observe quantum effects in the presence of classical noise that is much higher than the free-mass SQL.

Entanglement is an intriguing property of quantum systems where if the joint state of a two-state system is such that the state of each subsystem can be separately known, the joint state is separable. If, however, the joint state is a quantum superposition of (separable) states, and the two constituent states cannot be independently identified, the system is entangled. Prospects for producing and observing entanglement in macroscopic mechanical systems are promising [52-57]. If more than one carrier field is injected into the interferometer of Corbitt et al. [19,20], sidebands around the outgoing carrier fields are entangled, since all of them share the same modulation due to motion of the mirrors, which are, in turn, driven by each input sideband field. In the presence of the optical spring, the mechanical connection between the mirrors and the environment no longer dominates the dynamics, and the system is largely immune to thermal noise. The system can exhibit entanglement that is robust against classical noises. In fact, the entanglement generally exists as long as squeezing is available, which does not require going below the SQL [58].

\section{Macroscopic quantum mechanics}

If the total classical noise budget is below the SQL, the interferometer will be able to prepare macroscopic test masses into nearly quantum states, e. g., entangled states, and even states with non-positive-definite Wigner functions. Such tests can also be shown to be able to survive for a nontrivial amount of time, and then be verified by quantum tomography.

\subsection{Preparation of mechanical quantum states}

\subsubsection{Steady quantum states}

Damping associated with the optical spring does not bring the usual thermal noise, because the heat reservoir here has a very low effective temperature. This has proven to be an efficient means of cooling the translational motion degree of freedom of macroscopic test masses, some times referred to as "cold damping" [7, 8, 10-12,14]. Another type of cold damping is by measuring the motion of the test mass through the out-going optical field, and then use feedback control to suppress its motion.

In both types of cold damping, in the classical regime, this can be written as

$$
\frac{T_{\text {eff }}}{T_{0}}=\frac{Q}{Q_{0}}
$$

where the temperature, or thermal occupation number, is traded off against the quality factor of the mechanical oscillator. In the quantum regime, even in absence of classical noise, these types of damping each has its constraint. For radiation-pressure cooling alone, one needs the "resolved side-band limit", in which detuning of the optical cavity must be much larger than its bandwidth, while in the feedback cooling regime, measurement-induced decoherence must be appropriately avoided.

For mirrors suspended as pendulums, damping alone cannot be used to bring them into the quantum regime, due to the usually high levels of noise near pendulum resonances. For these objects, optical cooling and trapping is necessary. Optical cooling and trapping is achieved by a double optical spring, where one detuned optical field predominantly provides a restoring force, while another field with the opposite detuning mostly provides optical damping $[11,27]$. The final effective occupation number will be

$$
N=\frac{k_{B} T_{\text {eff }}}{\hbar \Omega_{\text {eff }}}, \quad \frac{T_{\text {eff }}}{T_{0}}=\frac{Q_{\text {eff }}}{Q_{0}} \frac{\Omega_{0}}{\Omega_{\text {eff }}}
$$

where $k_{B}$ and $\hbar$ are the Boltzmann and Planck constants, respectively, $T_{\text {eff }}$ is the temperature, and $\Omega_{\text {eff }}$ is the frequency of the oscillation mode. This allows a further suppression factor of $\left(\Omega_{\text {eff }} / \Omega_{0}\right)^{2}$. This technique has lead to the cooling of a macroscopic $2.5 \mathrm{~kg}$ effective mass (the differential translational mode of four $10 \mathrm{~kg}$ test masses) in the LIGO detector in Hanford, where an occupation of $\sim 200$ has been achieved [59].

\subsubsection{State preparation vs. state collapse}

Outgoing light from the optomechanical system usually contains information about the test mass, which means: (i) test mass quantum state is mixed with the state of light, and will not be pure, (ii) measurement of out-going light can project the test mass into pure states. A rule of thumb is that if the classical noise limits are below the free-mass SQL, then preparation of nearly pure quantum states are possible via 
state collapse $[60,61]$. The state prepared this way is not deterministic: depending on the measurement result, the test mass is projected into a different state. However, we can know this state, if noise budgets are known, and the system is a stable linear system, which will gradually forget its initial state, and with final state only determined by the measurement result and the noise budget. The formalism here have also been used to make connections to optimal control, and elaborate limits of feedback and radiation-pressure cooling [62].

\subsubsection{Mechanical entanglement}

Simply being nearly pure does not offer much insight into quantum mechanics. In linear systems driven by Gaussian optical states and classical noise, mirror states we prepare are going to be Gaussian. Nevertheless, interesting states can already be obtained, e. g., quantum entanglement.

The concept of entanglement originated from a gedankenexperiment by Einstein, Podolsky and Rosen (EPR) [63]. This experiment was set up to illustrate two interconnected and nonintuitive features of quantum mechanics - so nonintuitive that EPR take them as evidence that quantum mechanics is incomplete as a law of physics. The combination of these two features is called entanglement, and has been accepted as the defining feature of quantum mechanics.

The first feature is that, two quantum systems can be more similar than each of them can be specified individually. For example, for each of the two systems, we always have Heisenberg Uncertainty relation among the accuracies to which position and momentum can be described,

$$
\delta x_{1} \cdot \delta p_{1} \geq \hbar / 2, \quad \delta x_{2} \cdot \delta p_{2} \geq \hbar / 2
$$

but we are nevertheless allowed to have

$$
\delta\left(\frac{x_{1}-x_{2}}{\sqrt{2}}\right) \cdot \delta\left(\frac{p_{1}+p_{2}}{2}\right)<\hbar / 2
$$

which indicates a much higher level of correlation. The second feature is that this higher level of correlation can allow us, through making a quantum measurement, to influence the quantum state of an object which is at a spacelike separation, and therefore out of causal contact, from the measurement.

Optomechanical experiments with classical noise budgets below the free-mass SQL can also be used to generate entanglement among mechanical objects, as described in [60]. The idea is actually very much analogous to the original gedankenexperiment of EPR, in which two mechanical objects, well isolated from the environment, have their common and differential modes measured with different quantum-limited (i.e., classical noise below the SQL) schemes (e.g., in terms of signal recycling gain, readout quadrature, and input squeezing), in such a way that the differential mechanical mode is squeezed in position, and common mechanical mode squeezed in momentum - therefore realizing approximately an EPR state.

\subsection{Verification of mechanical quantum states}

\subsubsection{Wigner function}

In order to really demonstrate quantum mechanics, we must have the capability to measure the quantum state of the mechanical oscillator - more precisely, its density matrix. This can only be done if we repeatedly prepare the oscillator in the same state, and measure statistical distributions of linear combinations of position and momentum. If we know the distribution of

$$
X_{\zeta} \equiv \hat{x}(t) \cos \zeta+\frac{\hat{p}(t)}{m \Omega_{m}} \sin \zeta
$$

for each $\zeta$, this can be used to recover the Wigner function of the state, which in turn can yield the density matrix, through

$$
\rho\left(x, x^{\prime}\right)=\int e^{i p\left(x-x^{\prime}\right) / 2} W\left(\frac{x+x^{\prime}}{2}, p\right) d p .
$$

\subsubsection{Back-action-evading measurement of $X_{\zeta}$}

Although the distribution for $X_{\zeta}(t)$ we require is at an instantaneous value, measurement must be made over a nonzero period of time, because: (i) we only measure position directly, and therefore must allow some time to let the velocity distribution affect position distribution, and (ii) sensing noise for a infinitesimal data duration is infinite. This requires us to measure the position of the mirror continuously for a time interval, and use our knowledge of the device's dynamics to convert our measurement result into a measured value for a particular $X_{\zeta}(t)$. In addition, in order not to impose additional noise at the Heisenberg Uncertainty level, we need to design a different optimized scheme that is adapted to each quadrature we measure, instead of obtaining different quadrature information only through filtering data from the same device differently. One crucial approach to such an optimization is time-domain back-action evasion, achieved through measuring a time-dependent output light quadrature [64].

\section{Where are we experimentally?}

Over the last 20 years there have been many fixed mirror "benchtop" experiments performed as proof-of-principle demonstrations, or to develop and test control strategies. Configurations tested include power recycling [65], signal recycling [66], resonant sideband extraction [67], and signal recycling with variable reflectivity mirrors [32]. The use of squeezing to reduce shot noise has been verified at megahertz frequencies for power-recycled interferometers [68], for signal-recycled interferometers [69], for twin signalrecycling [70], in Sagnac interferometers [71], and in a suspended mirror signal recycled interferometer [72]. In [73] it was successfully demonstrated that a detuned filter cavity 
at the antisymmetric port of the interferometer can compensate for the dispersion of the detuned signal recycling cavity. The frequency response of speed meter configurations has been experimentally verified [75]. Power recycling has been implemented in LIGO [76], Virgo and GEO600. Signal recycling and RSE have been demonstrated on suspended interferometers including GEO600, the Caltech 40m prototype [22] and the Garching 30m prototype [77] interferometers.

We have seen that quantum optics can be used to modify the sensitivity of GW detectors in two ways: (1) By manipulating the quantum light states entering the interferometer (squeezed states); or (2) By direct dynamical modification of the internal quantum state via interaction with the mechanical system. To observe such effects requires the generation of highly squeezed states of light in the GW detection band (between $10 \mathrm{~Hz}$ and $10 \mathrm{kHz}$ ); the ability to manipulate the measurement quadrature; and an interferometer whose noise floor is limited by quantum radiation pressure noise at low frequencies and shot noise at high frequencies. In this section we will review progress in these areas. To date, no experimental observation of quantum radiation pressure noise or the standard quantum limit has been reported.

\subsection{Generation of squeezed light}

In the past seven years there has been tremendous progress in the development of squeezed light sources suitable for use in GW detectors [39] . Prior to 2004, squeezed light had not be produced at Fourier frequencies in the GW detection band below $10 \mathrm{kHz}$ and the squeezing factors observed were rather modest. Today we are close to the first implementation of this technique in a large scale GW detector.

Squeezing is produced by correlating pairs of quantum noise sidebands via the interaction of an intense laser field with a nonlinear medium [34]. One of the most successful approaches for continuous wave squeezed light generation is optical parametric oscillation (OPO). Common second-order nonlinear materials like $\mathrm{MgO}: \mathrm{LiNbO}_{3}$ and periodically poled potassium titanyl phosphate (PPKTP) can be used to produce broadband squeezing at the carrier wavelength of today's gravitational wave $(\mathrm{GW})$ detectors $(1064 \mathrm{~nm})$. A schematic layout of an squeezed light generation is shown in Fig. 17. A continuous-wave second harmonic light field (green light at $532 \mathrm{~nm}$ ) is focussed into the crystal which is placed inside a cavity [78]. Parametric downconversion of green photons produces pairs of correlated infra-red photons at $1064 \mathrm{~nm}$. When operated below threshold, a squeezed vacuum state is produced. The cavity can be either singly [78] or doubly resonant [79], increasing the parametric process. The squeezed states are detected by a balanced homodyne detector (HD) in which they are overlapped with an optical local oscillator field on a 50\%/50\% beam splitter. Note that for squeezed light injection into an interferometer, the local oscillator may also be provided by the weak laser field leaking out the antisymmetric port of the interferometer.

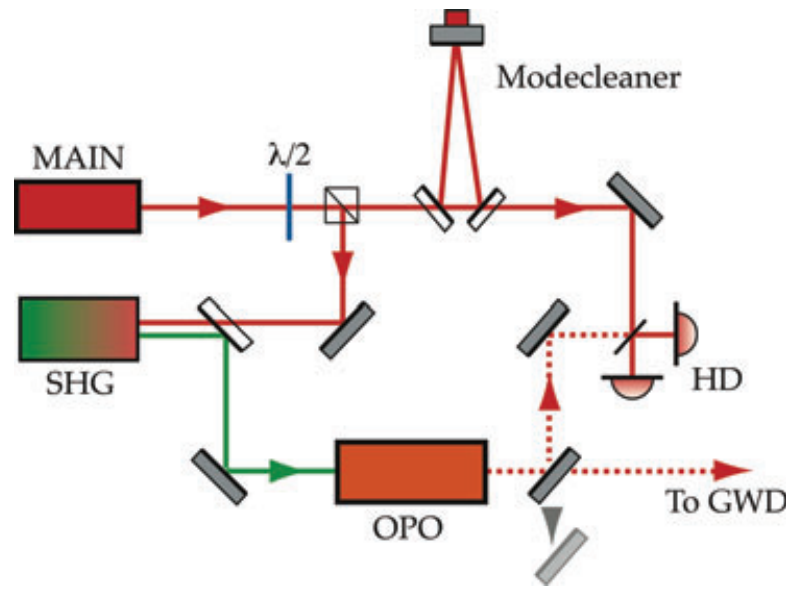

Figure 17 (online color at: www.lpr-journal.org) Schematic layout for squeezed light generation. Frequency double light from a second harmonic generator phase locked to the main red beam, is focussed into a nonlinear cavity to pump the OPO. The emerging squeezed vacuum, shown as the dotted red line, is directed either to a homodyne detector to measure the degree of squeezing or injected into the dark port of the gravitational wave detector (GWD). The main red beam is passed through a modecleaning cavity to match the spatial mode with that of the squeezed field, before being combined with the squeezed vacuum for the homodyne readout or sent off to the interferometer.

A necessary requirement for the application of squeezed states of light in GW detectors is the ability to generate squeezed states at audio-band Fourier frequencies. The first such demonstration was achieved in 2004 by McKenzie et al. [80] where a broadband squeezed field down to a few hundreds of Hertz was generated. Their breakthrough came with the realization that the dominant noise at audio frequencies that degrades squeezed light generation couples via the coherent laser field that was used to control the length of the squeezed light laser resonator and angle of squeezing. They avoided the use of a coherent locking beam at the signal frequency by using a technique known as quantum noise locking [81], where the inherent phase dependence of the generated squeezed state is used to provide the locking signal. Careful attention must also be paid to avoid noise coupling via scattered light.

In an improved experiment in 2007, Vahlbruch et al. [82, $83]$ observed squeezing down to $1 \mathrm{~Hz}$. A highly stable coherent control scheme was demonstrated in $[73,74]$. It relies on a coherent control field that is frequency shifted by several $\mathrm{MHz}$ with respect to the squeezed field and that is also parametically amplified by the squeezed light source thereby giving a phase reference for the parametric process. Scattered and frequency shifted photons were identified to be a major noise source at low frequencies in previous and current experiments with OPO squeezers. Figure 18 shows the current best performance by a squeezer in the audio $\mathrm{GW}$ detection [84].

Injection of squeezed states into the output signal port of an interferometer leads to a sensitivity increase equivalent to a laser power increase of exactly the same factor. Note, that optical power (photon) loss $\left(t^{2}\right)$ increases the value 


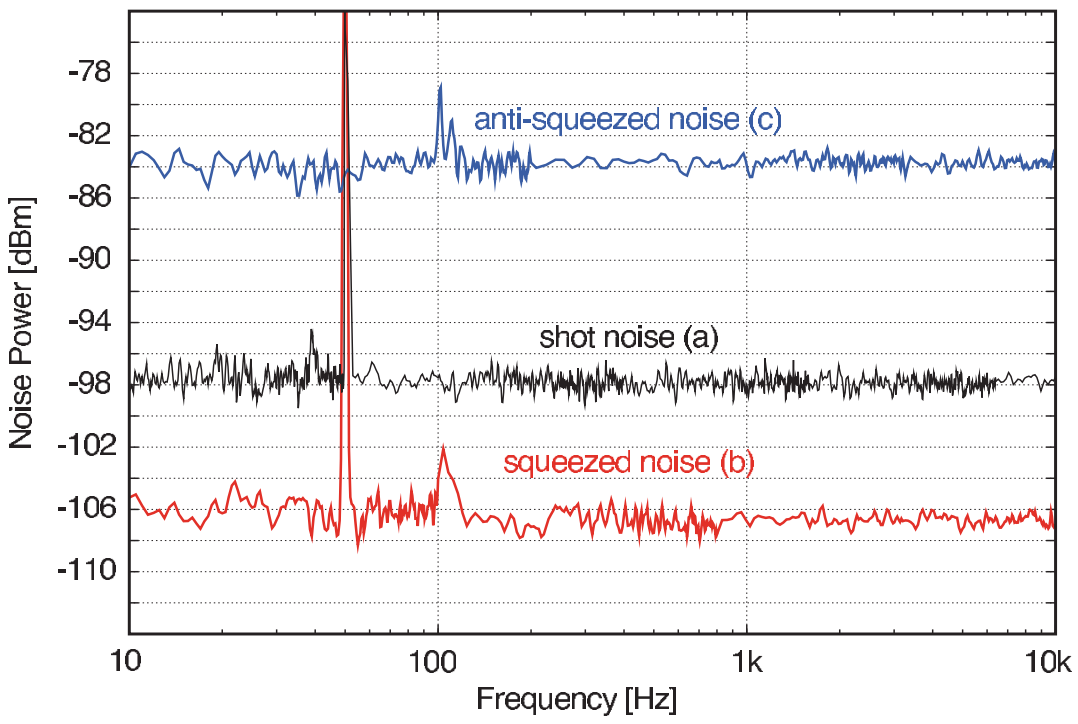

Figure 18 (online color at: www.lprjournal.org) From [84]. Trace (a) shows the vacuum noise level of the homodyne detector. Trace (b) shows the noise powers of squeezed states measured with the same detector setting. Squeezed vacuum noise was observed throughout the complete spectrum from $10 \mathrm{~Hz}$ to above $10 \mathrm{kHz}$. A nonclassical noise suppression of up to $9 \mathrm{~dB}$ below vacuum noise was observed. Reproduced from [84], Fig. 4. for the squeezed variance according to $V_{1}^{\prime}=V_{1}\left(1-t^{2}\right)+t^{2}$. For future $\mathrm{GW}$ detectors the reduction of optical loss will therefore be increasingly important.

\subsection{Squeezed light enhanced suspended interferometers}

With suitable squeezers now becoming available, programs have been put into place to demonstrate the experimental compatibility of squeezing with low noise suspended interferometry. Goda et al. injected squeezed states into a prototype gravitational wave detector and showed that a system consisting of a squeezed light input and an interferometer with suspended test masses can be sufficiently controlled in order to gain a nonclassical sensitivity improvement [72]. Whilst an important demonstration for establishing the compatibility of squeezing enhancement with suspended-mirror interferometer dynamics, this experiment showed modest squeezing enhancement at frequencies above the GW band and in a low sensitivity instrument.

To fully develop a squeeze injection source that is suitable for long baseline GW detectors, experimental programs to inject squeezing into one of the $4 \mathrm{~km}$ long interferometers of LIGO and into GEO600 are underway. The LIGO experiment will test squeezed state injection down to $100 \mathrm{~Hz}$ and will deploy an OPO geometry which should be less sensitive to back scattered light $[79,85]$.

\subsection{Towards the SQL}

To date, no optical experiment limited by quantum radiation pressure noise on a "free mass" has been built necessarily implying that the SQL has yet to be observed in such optomechanical systems. There are a number of projects underway at Massachussetts Institute of Technology, in the USA; the National Astronomical Observatory Japan, the Albert Einstein Institute, Hannover, Germany, and in Australia at The Australian National University and the University of
Western Australia, using optomechanical systems spanning several mass scales. The effort is driven by the desire to build more and more sensitive detectors, to prove technologies for future generation $\mathrm{GW}$ detectors, to produce ponderomotive squeezing, and to achieve the goal of cooling macroscopic mechanical systems to their quantum ground states.

Most of these experiments are predicated on a common ingredient: an optical cavity comprising one large and easily actuated mirror (for control) and a light mirror oscillator of ultra high quality factor minimizing thermal noise, isolated from vibrational forces sufficiently well that it responds predominantly to radiation pressure forces. System parameters are chosen such that thermal noise rolls off faster with frequency than quantum radiation pressure noise, which should enable the SQL to be observed in the $100 \mathrm{~Hz}$ to few $\mathrm{kHz}$ band. Figure 19 shows the predicted noise budget for a $10 \mathrm{~m}$ baseline SQL interferometer under construction at the Albert Einstein Institute in Germany [86] . In principle, the biggest impediment to reaching the SQL comes from coating thermal noise [5]. In practice, attempts to operate such optomechanical systems in the quantum regime have been impeded by other noise sources such as thermal noise from the attachment points of the suspended mirrors [87], and instabilities such as torque instabilities [88].

In the absence of experimental systems dominated by quantum radiation pressure noise, the physics behind the quantum effects these systems seek to observe has been tested using classical analogs [89], [90]. In such systems, a cavity with a movable mirror is driven by amplitude and phase modulated fields used to mimic quantum correlations. For example, using the layout shown in Fig. 20, Mow-Lowry et al. [90], imposed broadband amplitude (AM) and phase modulation (PM) onto the carrier. The radiation pressure force from the AM moved the mirror resulting in the conversion of AM into PM. Adjusting the noise used to drive one of the modulators provided a frequency independent variable phase shift. Adjustment of this phase shift caused the two modulation (noise) sources to add destructively as shown in Fig. 21, mimicking beating the SQL. For details, see [90]. 


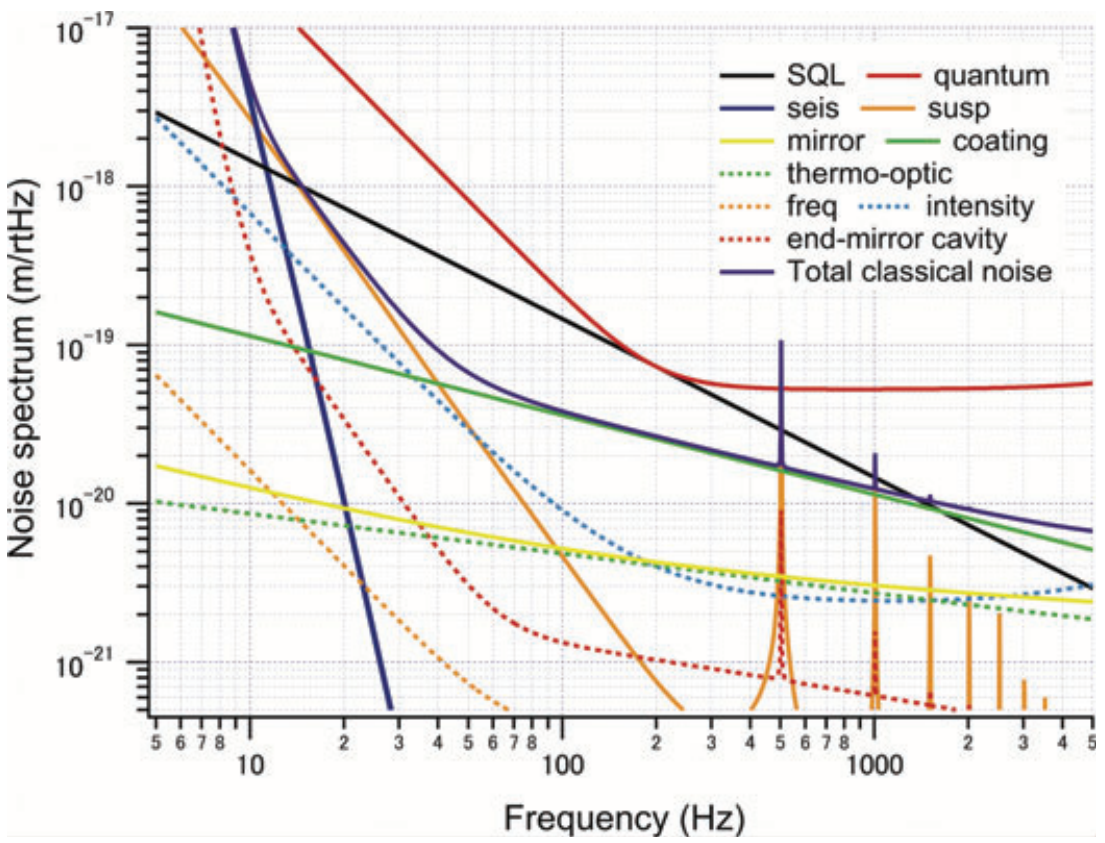

Figure 19 (online color at: www.lprjournal.org) Anatomy of the predicted noise floor for the AEI $10 \mathrm{~m}$ interferometer. The various noise contributions include seis: seismic noise; mirror: mirror thermal noise; thermo-optic: thermo optic thermal noise; freq: laser frequency noise; susp: suspension thermal noise; coating: coating thermal noise; intensity: laser intensity noise; and quantum: total quantum noise. Courtesy Stefan Goler; similar to Fig. 3, in [86].

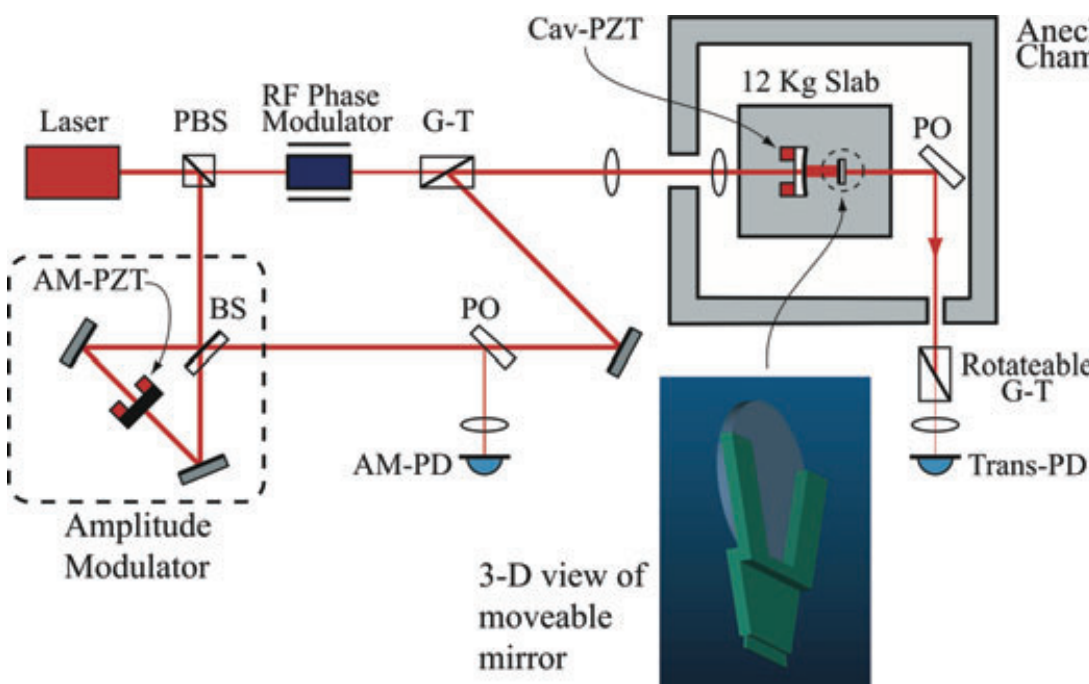

Figure 20 (online color at: www.lprjournal.org) A schematic diagram of the optical layout used in [90]. A close-up view of the radiation pressure driven flexure-mirror oscillator is also shown. PBS, polarizing beam splitter; BS, beam splitter; G-T, GlanTaylor polarizer; PO, pickoff mirror; PD, photodetector. Reproduced from [90], Fig. 2.

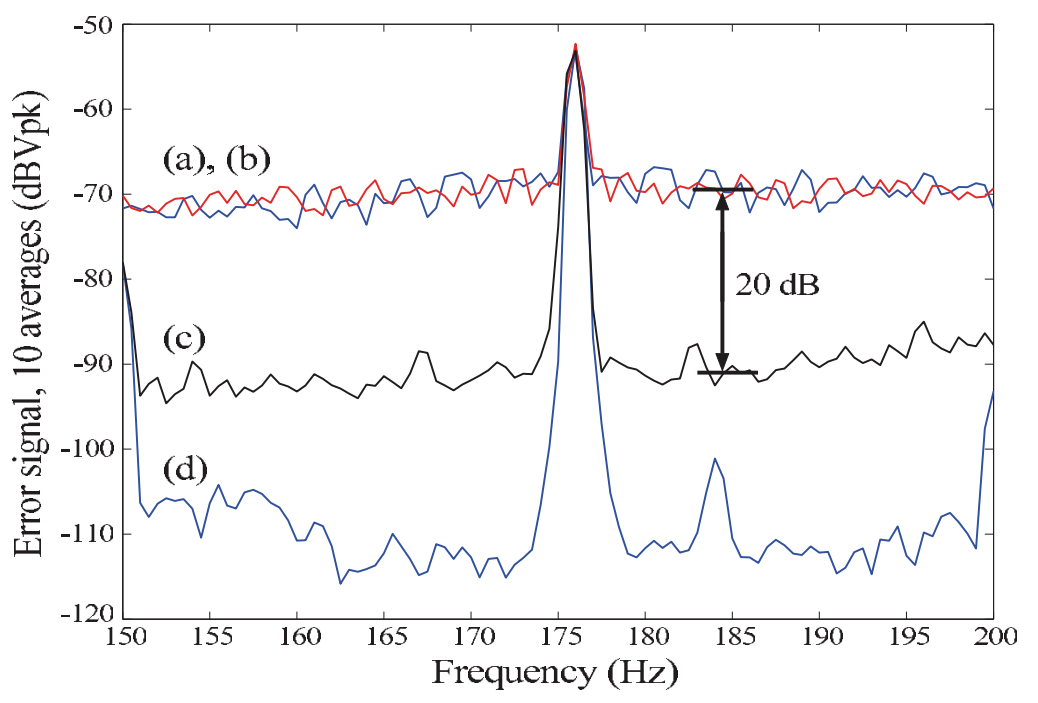

Figure 21 (online color at: www.lpr-journal.org) Results from the experiment depicted in Fig. 20. Traces (a) and (b) show the excess noise added first through the amplitude modulator and second through the frequency modulator. Trace (c) shows the noise, originating from the two orthogonal quadratures, cancelling to improve the signal to noise ratio by approximately a factor of 10 . Trace (d) is the background noise, recorded with no optical modulations added in the frequency band shown. The spike, at $176 \mathrm{~Hz}$, was a signal injected through the cavity PZT. Reproduced from [90], Fig. 4. 


\subsection{The optical spring and optical cooling}

We have already established the central role of the optical spring in the optomechanical systems of GW detectors, both for resonant enhancement of detector sensitivity, and for quantum state preparation of the interferometer mirrors. Preliminary evidence for observation of the optical spring effect was first report by Dorsel et al. in 1983 [91]. The first definitive observation of an optical spring mediated resonance shift was reported by Sheard et al. in 2004 [18], where a frequency shift of a few $\mathrm{Hz}$ was observed corresponding to an optical spring stiffness of $150 \mathrm{~N} / \mathrm{m}$. Corbitt et al. [92] demonstrated the optical spring effect using the optomechanical system shown in Fig. 22. The $250 \mathrm{~g}$ input mirror of a $0.9 \mathrm{~m}$ long cavity was suspended as a pendulum with oscillation frequency of $1 \mathrm{~Hz}$ for the longitudinal mode. The $1 \mathrm{~g}$ end mirror was suspended by two optical fibers 300 micron in diameter, giving a natural frequency $172 \mathrm{~Hz}$ for its mechanical mode, with quality factor of 3200 . In this experiment, the radiation pressure force completely dominated the mechanical forces on the 1 gram mirror shifting its frequency to $\sim 5 \mathrm{kHz}$ (see Fig. 23). This frequency shift corresponds to a stiffness of $2 \times 10^{6} \mathrm{~N} / \mathrm{m}$; the optical spring provided greater stiffness than if the optical mode of the cavity were replaced with a diamond rod of the same dimensions. For details, see [92]. The optical spring effect in a signal recycled interferometer was observed by Miyakawa et al. [22] using the $40 \mathrm{~m}$ prototype interferometer at Catech.

By modifying the delay between the radiation pressure force and the mechanical response, the optical force can be made viscous, enabling damping of the motion. Using

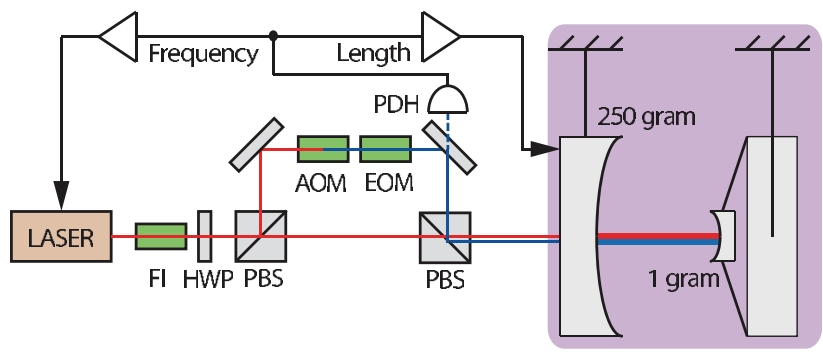

Figure 22 Schematic of the experimental layout of the MIT optical spring experiment [92]. Highly frequency- and intensity-stabilized laser light is incident on a cavity where the $1 \mathrm{gram}$ end mirror is suspended as a pendulum. An acoustooptic modulator (AOM) frequency-shifts about $10 \%$ of the light by one free spectral range of the cavity. The two optical fields can be independently detuned to create an optical spring and to provide optical damping. An electrooptic modulator (EOM) applies phase modulation sidebands used generate a Pound-Drever-Hall (PDH) signal for locking the cavity to the laser frequency. FI refers to a Faraday isolator. Reproduced from [11], Fig. 1.

cavity optical delay and electronic feedback control, Corbitt et al. [92] have cooled the pendular oscillation mode of a gram scale oscillator from room temperature to $6.9 \mathrm{mK}$ (see Fig. 24). Recently, Wipf et al. [93] have cooled a gram-scale oscillator to $0.8 \mathrm{mK}$, corresponding to 35000 phonon in the oscillator mode. One of the great advantages of this approach is that when the optical spring dominates the mechanical spring, the oscillator is decoupled from the thermal

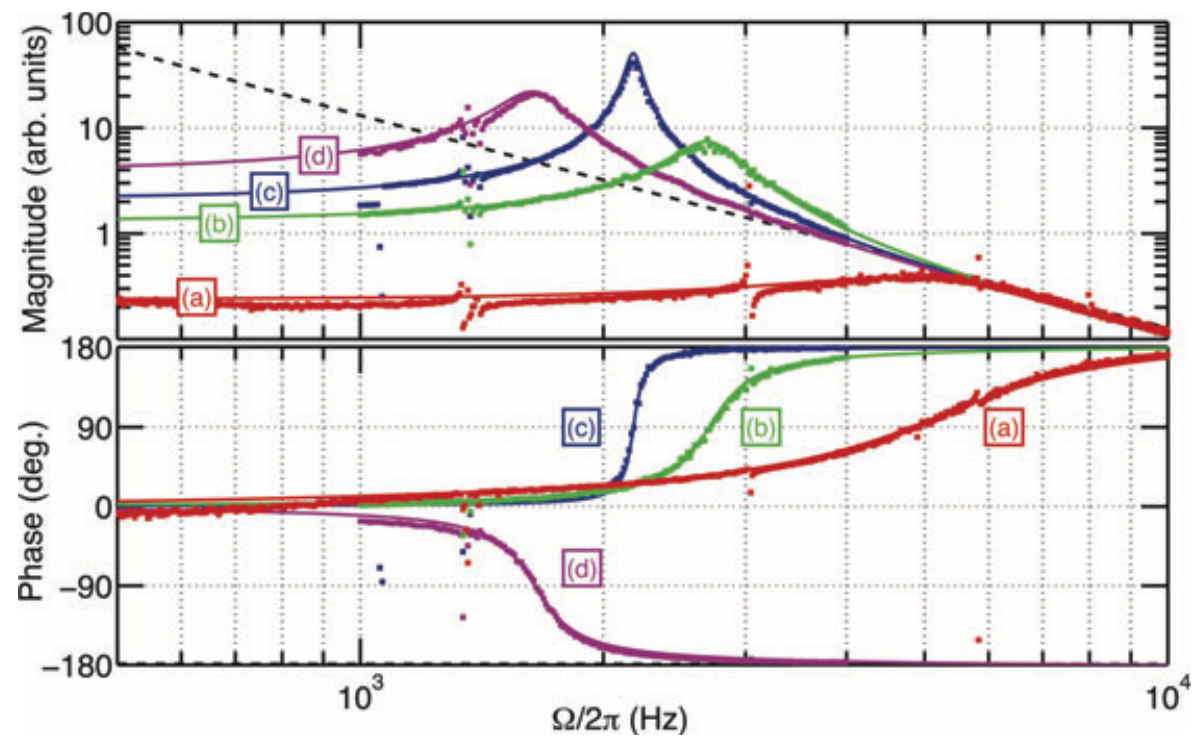

Figure 23 (online color at: www.lpr-journal.org) Frequency response of the optomechanical oscillator of Fig. 22, as a function of cavity detuning of a strong optical field, showing increasingly stronger optical springs, with the resonance shifted up to $5 \mathrm{kHz}$. A weaker optical field detuned to the opposite side provided an optical damping force that stabilized the optical spring in curve (d), thus making an all-optical trap for the 1 gram mirror. Measured transfer functions of displacement per force are shown as points, while the solid lines are theoretical curves. The dashed line shows the response of the system with no optical spring. An unstable optical spring resonance with varying damping and resonant frequency is produced when (a) $\delta_{c}=0.5 \gamma, \delta_{s c}=0$; (b) $\delta_{c}=3 \gamma, \delta_{s c}=0.5 \gamma$; (c) $\delta_{c}=3 \gamma, \delta_{s c}=0$; and it is stabilized in (d) $\delta_{c}=3 \gamma, \delta_{s c}=-0.3 \gamma$; where $\delta_{c}$ is the carrier detuning, $\delta_{s c}$ is the sub-carrier detuning, and $\gamma$ is the resonant linewidth. Reproduced from [11], Fig. 3. 


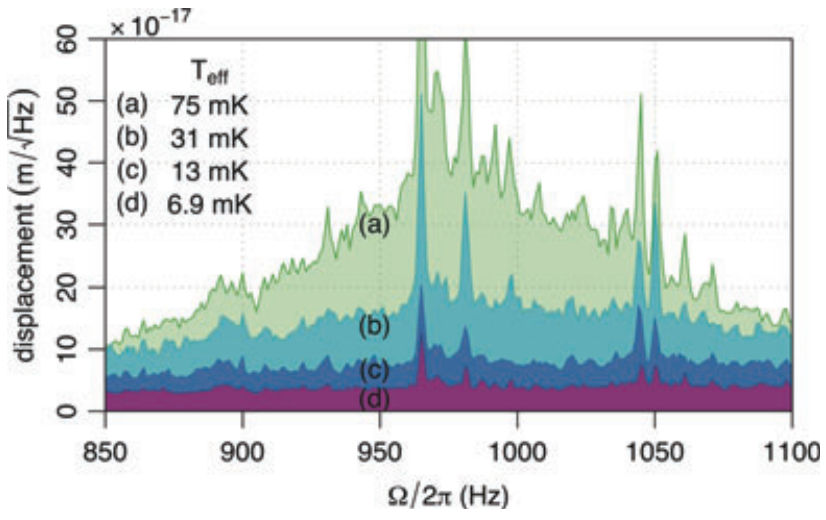

Figure 24 (online color at: www.lpr-journal.org) Optical cooling data from the MIT experiment showing cooling of the peak to 6.9 mK. Reproduced from [11], Fig. 3.

environment and, therefore, decoherence times should be much longer (see Sect. 7).

Mow-Lowry et al. [94] introduced the required delay electronically via the feedback loop controlling the cavity locking. They reported cooling from room temperature to $70 \mathrm{mK}$. Using a similar technique, but applied to electronic feedback modification of the mechanical suspension "spring" supporting the LIGO mirrors, Abbott et al. [59] reported cooling the mechanical resonator to $1.4 \mu \mathrm{K}$, corresponding to a mere 234 quanta in the longitudinal mode of a mechanical oscillator with effective mass of $2.5 \mathrm{~kg}$ (see Sect. 7).

\section{Summary}

Laser interferometry has come a long way since Weiss [3] and Forward [4] first proposed its use for GW detection. The humble Michelson interferometer now forms the platform for a host of concepts including power recycling, signal recycling and resonant sideband extraction. Instruments are being built with sensitivities limited by the quantum nature of light spawning new ideas and concepts in quantum metrology and quantum non demolition such as squeezing, variational readouts and speed meters. The optical force plays a central role in such systems leading to SQL beating opto-mechanical resonances and the possibility of optical cooling mechanical resonators to quantum ground states.

In this article we have reviewed the major ideas in laser interferometry for gravitational wave detection and briefly introduced the quantum formalism used to analyze such systems. We examined the connections with quantum optomechanics and macroscopic quantum mechanics. We brought together the state-of-the-art as of 2010 in experimental verification and the quest to reach and then breach the SQL.

Future generation gravitational wave detectors are predicted to reach the SQL with $40 \mathrm{~kg}$ scale test masses. When the SQL is reached this massive mechanical system will exist in the ground state of the center of mass motion of the optomechanical oscillator. Observation of quantum mechanical weirdness on truly massive human scales will be within reach. Alternatively, new physics will have intervened to mask the quantum from the classical world. Even as these giant detectors set off a revolution in gravitational wave astronomy, these exquisitely sensitive devices may also revolutionize our understanding of quantum mechanics on unprecedentedly large scales.

Acknowledgements. This research was supported by the Australian Research Council, the National Science Foundation and the Deutsche Forschungsgemeinschaft through the Centre for Quantum Engineering and Space-Time Research, QUEST. The authors would like to acknowledge and thank the many gifted past and present researchers from their respective groups.

Received: 5 November 2010, Revised: 26 January 2011, Accepted: 16 February 2011

Published online: 14 March 2011

Key words: Gravitational waves, quantum optics, opto-mechanics.

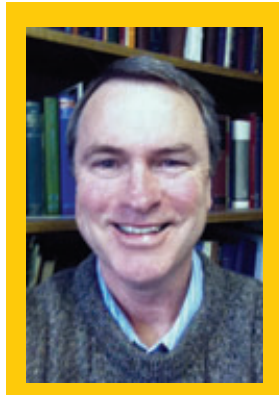

David E. McClelland received his Ph. D. degree from the University of Otago, New Zealand in 1987. He was awarded a Beverly Research Fellowship before being appointed in 1988 to The Australian National University (ANU), Canberra, Australia, as lecturer. He is now Professor and Head of the Department of Quantum Science in the Research School of Physics and Engineering, and Director of The ANU Centre for Gravitational Physics. His research interests include the development of optical techniques for laser-interferometer-based gravitational wave detectors, the generation and application of squeezed states of light and quantum-opto mechanics.

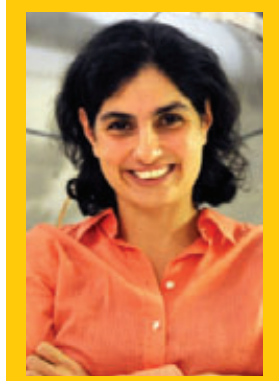

Nergis Mavalvala received a Ph. D. in physics from the Massachussetts Institute of Technology (MIT) in 1997, and a B. A. in physics and astronomy from Wellesley College in 1990. After completing a postdoctoral associateship and a research scientist position at the California Institute of Technology, she joined the Physics faculty at MIT in January 2002 She is now Professor of Physics. Her primary research has been in instrument development for interferometric gravitational-wave detectors, and the development of techniques for quantum noise reduction and quantum-opto mechanics.

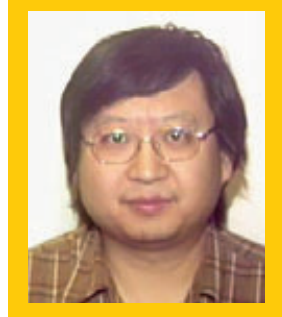

Yanbei Chen completed his Ph. D. at the California Institute of Technology in 2003. He worked as a postdoctoral fellow and then junior research group leader at the Max Planck Institute for Gravitational Physics (also known as the AlbertEinstein Institute, or the AEI) in Golm, Germany, before re- joining Caltech in 
2007 as an Associate Professor. His work spans: applications of quantum measurement theory to laser interferometer gravitational-wave detectors; macroscopic quantum mechanics of kg-scale Test Masses; novel approaches to gravitationalwave detection; gravitational-wave sources and general relativity and strategies for analyzing gravitational-wave data.

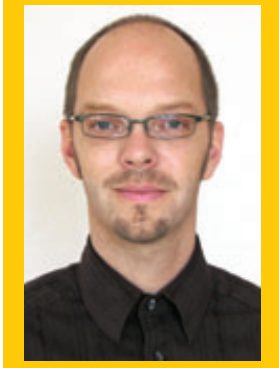

Roman Schnabel studied physics at the University of Hannover, Germany. After his Ph. D. he was awarded the FeodorLynen fellowship of the Alexander-vonHumboldt Foundation for a postdoctoral position at the Australian National University. His work spans laser spectroscopy on trapped ions, quantum teleportation, and squeezed light for the detection of gravitational waves. He is now Professor at the Leibniz Universität Hannover and a member of QUEST.

\section{References}

[1] A. Einstein, Sitz.ber. Preuss. Akad. Wiss. 142 (1917).

[2] L. Ju, D. G. Blair, and C. Zhao, C. Rep. Prog. Phys. 631317 (2000).

[3] R. Weiss, Quarterly Progress Report, Research Laboratory of Electronics, MIT 105: 54 (1972).

[4] R. Forward, Phys. Rev. D 17, 379 (1978).

[5] G. Harry and the LIGO Scientific Collaboration, Class. Quantum Grav. 27, 084006 (2010).

[6] V. B. Braginsky and F. Y. Khalili, in: Quantum Measurement, edited by K. S. Thorne (Cambridge University Press, Cambridge, 1992).

[7] D. Kleckner and D. Bouwmeester, Nature 444, 75 (2006).

[8] S. Gigan, H. Böhm, M. Paternostro, F. Blaser, G. Langer, J. Hertzberg, K. Schwab, D. Bäuerle, M. Aspelmeyer, and A. Zeilinger, Nature 444, 67 (2006).

[9] O. Arcizet, P.-F. Cohadon, T. Briant, M. Pinard, and A. Heidmann, Nature 444, 71 (2006).

[10] A. Schliesser, P. Del'Haye, N. Nooshi, K. J. Vahala, and T. J. Kippenberg, Phys. Rev. Lett. 97, 243905 (2006).

[11] T. Corbitt, Y. Chen, E. Innerhofer, H. Müller-Ebhardt, D. Ottaway, H. Rehbein, D. Sigg, S. Whitcomb, C. Wipf, and N. Mavalvala, Phys. Rev. Lett. 98, 150802 (2007).

[12] J. G. E. Harris, B. M. Zwickl, and A. M. Jayich, Rev. Sci. Instrum. 78, 013107 (2007).

[13] M. Poggio, C. L. Degen, H. J. Mamin, and D. Rugar, Phys. Rev. Lett. 99, 017201 (2007).

[14] T. Caniard, P. Verlot, T. Briant, P.-F. Cohadon, and A. Heidmann, Phys. Rev. Lett. 99, 110801 (2007).

[15] C. H. Metzger and K. Karrai, Nature 432, 1002 (2004).

[16] A. Buonanno and Y. Chen, Phys. Rev. D 65, 042001 (2002).

[17] V. B. Braginsky and S. P. Vyatchanin, Phys. Lett. A 293, 228 (2002).

[18] B. S. Sheard, M. B. Gray, C. M. Mow-Lowry, D. E. McClelland, and S. E. Whitcomb, Phys. Rev. A, 69, 051801 (2004).

[19] T. Corbitt, Y. Chen, S. Whitcomb, and N. Mavalvala, Phys. Rev. A, 72, 013818 (2005).

[20] T. Corbitt, Y. Chen, F. Khalili, D. Ottaway, S. Vyatchanin, S. E. Whitcomb, and N. Mavalvala, Phys. Rev. A 73, 023801 (2006).
[21] T. Corbitt, D. Ottaway, E. Innerhofer, J. Pelc, and N. Mavalvala, Phys. Rev. A 74, 021802 (2006).

[22] O. Miyakawa, R. Ward, R. Adhikari, M. Evans, B. Abbott, R. Bork, D. Busby, J. Heefner, A. Ivanov, M. Smith, R. Taylor, S. Vass, A. Weinstein, M. Varvella, S. Kawamura, F. Kawazoe, S. Sakata, and C. Mow-Lowry, Phys. Rev. D 74, 022001 (2006).

[23] P. Fritschel, Proc. SPIE 4856-39, 282 (2002).

[24] L. D. Fiore and the VIRGO Collaboration, Class. Quantum Grav. 19, 1421 (2002).

[25] B. Willke and the GEO Collaboration, Class. Quantum Grav. 19,1377 (2002).

[26] A. Abramovici, W. E. Althouse, R. W. P. Drever, Y. Gürsel, F. J. Raab, D. Shoemaker, L. Sievers, R.E. Spero, K. S. Thorne, R. E. Vogt, R. Weiss, S. E. Whitcomb, and M. E. Zucker, Science 256, 325 (1992).

[27] G. Cagnoli, J. Hough, D. DeBra, M. M. Fejer, E. Gustafson, S. Rowan, and V. Mitrofanov, Phys. Lett. A 272, 39 (2000); N. Robertson, Class. Quantum Grav. 17, R19 (2000).

[28] R. W. P. Drever, in: Gravitational Radiation, edited by N. Deruelle and T. Piran (North Holland, Amsterdam, 1983), pp. 321-338.

[29] B. Willke, Laser Photonics Rev. 4, 780 (2010).

[30] B. J. Meers, Phys. Rev. D 38, 2317 (1988).

[31] H. Lück et al, Class. Quantum Grav. 23, S71-S78 (2006).

[32] G. de Vine, D. A. Shaddock, and D. E. McClelland, Optics Letters 271507 (2002); K. A. Strain and J. Hough, Rev. Sci. Instrum. 65, 799 (1994)

[33] J. Mizuno, K. A. Strain, P. G. Nelson, J. M. Chen, R. Schilling, A. Rüdiger, W. Winkler, and K. Danzmann, Phys. Lett. A 175, 273 (1993).

[34] H. A. Bachor and T.C. Ralph, A Guide to Experiments in Quantum Optics (Wiley-VCH, Weinheim, 2004) and references therein.

[35] C. C. Gerry and P. L. Knight, Introductory Quantum Optics (Cambridge University Press, Cambridge, UK, 2004).

[36] C. M. Caves, Phys. Rev. Lett. 45, 75 (1980).

[37] C. M. Caves, Phys. Rev. D 23, 1693 (1981).

[38] P. R. Saulson, Fundamentals of Interferometrtic Gravitational Wave Detection (World Scientific, Singapore, 1998).

[39] R. Schnabel, N. Mavalvala, D. E. McClelland, and P. K. Lam, Nat. Commun. 1, Article number: 121 (2010), DOI: $10.1038 /$ ncomms 1122 .

[40] W. G. Unruh, in: Quantum Optics, Experimental Gravitation, and Measurement Theory, edited by P. Meystre and M. O. Scully (Plenum, New York, 1983), pp. 647-660.

[41] H. P. Yuen, Phys. Rev. Lett. 51, 719-722 (1983).

[42] M. T. Jaekel and S. Reynaud, Europhys. Lett. 13, 301 (1990).

[43] H. J. Kimble, Y. Levin, A. B. Matsko, K. S. Thorne, and S. P. Vyatchanin, Phys. Rev. D 65, 022002 (2002).

[44] P. Purdue and Y. Chen, Phys. Rev. D 66, 122004 (2002).

[45] J. Harms, Y. Chen, S. Chelkowski, A. Franzen, H. Vahlbruch, K. Danzmann, and R. Schnabel, Phys. Rev. D 68, 042001 (2003).

[46] T. Corbitt, N. Mavalvala, and S. E. Whitcomb, Phys. Rev. D. 70, 022002 (2004).

[47] F. Y. Khalili, Phys. Rev. D 77 , 062003 (2008).

[48] V. B. Braginsky and F. Y. Khalili, Rev. Mod. Phys. 68, 1, (1996)

[49] P. Purdue, Phys. Rev. D 66, 022001 (2002).

[50] Y. Chen, Phys. Rev. D 67, 122004 (2003). 
[51] A. Buonanno and Y. Chen, Phys. Rev. D 64, 042006 (2001).

[52] S. Mancini, V. Giovannetti, D. Vitali, and P. Tombesi, Phys. Rev. Lett. 88, 1204012002.

[53] S. Pirandola, S. Mancini, D. Vitali, and P. Tombesi, J. Opt. B Quantum. Semiclassical. Opt. 5, S523 (2003).

[54] S. Mancini, D. Vitali, V. Giovannetti, and P. Tombesi, Eur. Phys. J. D 22, 417 (2003).

[55] S. Giannini, S. Mancini, and P. Tombesi, Quantum Inform. Comput. 3, 265 (2003).

[56] S. Pirandola, D. Vitali, P. Tombesi, and S. Lloyd, Phys. Rev. Lett. 97, 150403 (2006).

[57] D. Vitali, S. Gigan, A. Ferreira, H. R. Böhm, P. Tombesi, A. Guerreiro, V. Vedral, A. Zeilinger, and M. Aspelmeyer, Phys. Rev. Lett. 98, 030405 (2007).

[58] C. Wipf, T. Corbitt, Y. Chen, and N. Mavalvala, New J. Phys. 10, 095017 (2008).

[59] B. Abbott, R. Abbott, R. Adhikari, and 441 others (LIGO Scientific Collaboration), New J. Phys. 11, 073032 (2009).

[60] H. Müller-Ebhardt, H. Rehbein, R. Schnabel, K. Danzmann, and Y. Chen, Phys. Rev. Lett. 100, 013601 (2008).

[61] H. Müller-Ebhardt, H. Rehbein, C. Li, Y. Mino, K. Somiya, R. Schnabel, K. Danzmann, and Y. Chen, Phys. Rev. A 80 043802 (2009).

[62] H. Miao, S. L. Danilishin, H. Müller-Ebhardt, and Y. Chen, New J. Phys. 12, 083032 (2010).

[63] A. Einstein, B. Podolsky, and N. Rosen, Phys. Rev. 47777 (1935).

[64] H. Miao, S. Danilishin, H. Müller-Ebhardt, H. Rehbein, K. Somiya, and Y. Chen, Phys. Rev. A 81012114 (2010).

[65] M. Ando, K. Arai, K. Kawabe, and K. Tsubono, Physics Letters A 248, 145 (1998); D. Sigg, N. Mavalvala, J. Giaime, P. Fritschel, and D. Shoemaker, Applied Optics 37, 5687 (1998); M. W. Regehr, F. J. Raab, and S. E. Whitcomb, Opt. Lett. 20, 1507 (1995).

[66] M. B. Gray, A. J. Stevenson, H.-A. Bachor, and D. E. McClelland, Applied Optics 37, 5886 (1998); K. A. Strain and B. J. Meers, Phys. Rev. Lett. 66, 1391 (1991).

[67] K. A. Strain, G. Muller, T. Delker, D. H. Reitze, D. B. Tanner, J.E. Mason, P. Willems, D. Shaddock, M. B. Gray, C. Mow-Lowry, and D. E. McClelland, Appl. Opt. 42, 1244 (2003).

[68] K. McKenzie, D. A. Shaddock, D.E. McClelland, B. C. Buchler, and P. K. Lam, Phys. Rev. Lett. 88, 231102 (2002).

[69] H. Vahlbruch, S. Chelkowski, B. Hage, A. Franzen, K. Danzmann, and R. Schnabel, Phys. Rev. Lett. 95, 211102 (2005).

[70] A. Thüring, C. Graef, H. Vahlbruch, M. Mehmet, K. Danzmann, and R. Schnabel, Opt. Lett. 34824 (2009).

[71] T. Eberle, S. Steinlechner, J. Bauchrowitz, V. Handchen, H. Vahlbruch, M. Mehmet, H. Mueller-Ebhart, and R. Schnabel, Phys. Rev. Lett 104251102 (2010).

[72] K. Goda, O. Miyakawa, E. E. Mikhailov, S. Saraf, R. Adhikari, K. McKenzie, R. Ward, S. Vass, A. J. Weinstein, and N. Mavalvala, Nature Phys. 4, 472 (2008).

[73] H. Vahlbruch, S. Chelkowski, B. Hage, A. Franzen, K. Danzmann, R. Schnabel, Class. Quantum Grav. 23 S251 (2006).
[74] S. Chelkowski, H. Vahlbruch, K. Danzmann, and R. Schnabel, Phys. Rev. A 75, 043814, (2007).

[75] G. de Vine, M. B. Gray, D. E. McClelland, Y. Chen, and S. E. Whitcomb, Phys. Lett. A 316, 17 (2003).

[76] B. Abbott, R. Abbott, R. Adhikari, and 366 others, Nucl. Instrum. Methods Phys. Res. A 517, 154 (2004).

[77] G. Heinzel, J. Mizuno, R. Schilling, W. Winkler, A. Rüdiger, and K. Danzmann, Phys. Lett. A 217305 (1996).

[78] P. K. Lam, T. C. Ralph, B. C. Buchler, D. E. McClelland, H.-A. Bachor, and J. Gao, J. Opt. B, Quantum Semiclass. Opt. 1, 469 (1999).

[79] K. McKenzie, M. B. Gray, S. Gossler, P. K. Lam, and D. E. McClelland, Class. Quantum Grav. 23, S245-S250 (2006).

[80] K. McKenzie, N. Grosse, W. P. Bowen, S. E. Whitcomb, M. B. Gray, D. E. McClelland, and P. K. Lam, Phys. Rev. Lett. 93, 161105 (2004).

[81] K. McKenzie, E.E. Mikhailov, K. Goda, P. K. Lam, N. Grosse, M. B. Gray, N. Mavalvala, and D. E. McClelland, J. Opt. B, Quantum Semiclassical Opt. 7, S421 (2005).

[82] H. Vahlbruch, S. Chelkowski, K. Danzmann, and R. Schnabel, New J. Phys. 9, 371, (2007).

[83] H. Vahlbruch, M. Mehmet, S. Chelkowski, B. Hage, A. Franzen, N. Lastzka, S. Goßler, K. Danzmann, and R. Schnabel, Phys. Rev. Lett. 100, 033602 (2008).

[84] H. Vahlbruch, A. Khalaidovski, N. Lastzka, C. Gräf, K. Danzmann, and R. Schnabel, Class. Quantum Grav. 27, 084027 (2010).

[85] M. Stefszky, C. M. Mow-Lowry, K. McKenzie, S. Chua, B. C. Buchler, T. Symul, D. E. McClelland, and P. K. Lam, J. Phys. B, At. Mol. Opt. Phys. 44, 015502, (2011).

[86] http://iopscience.iop.org/0264-9381/27/8/084023.

[87] N. Mavalvala, Private Communication (2010).

[88] S. Kawamura, Private Communication (2010).

[89] T. Caniard, P. Verlot, T. Briant, P.-F. Cohadon, and A. Heidmann, Phys. Rev. Lett. 99, 110801 (2007); P. Verlot, A. Tavernarakis, T. Briant, P.-F. Cohadon, and A. Heidmann, Phys. Rev. Lett. 102, 103601 (2009); F. Marino, F. S. Cataliotti, A. Farsi, M. Siciliani de Cumis, and F. Marin, Phys. Rev. Lett. 104, 073601 (2010).

[90] C. M. Mow-Lowry, B.S. Sheard, M. B. Gray, D. E. McClelland, and S. E. Whitcomb, Phys. Rev. Lett. 92, 161102 (2004).

[91] A. Dorsel, J. D. McCullen, P. Meystre, E. Vignes, and H. Walther, Phys. Rev. Lett. 51, 1550 (1983).

[92] T. Corbitt, C. Wipf, T. Bodiya, D. Ottaway, D. Sigg, N. Smith, S. E. Whitcomb, and N. Mavalvala, Phys. Rev. Lett. 99, 160801 (2007).

[93] C. Wipf, T. Bodiya, T. Corbitt, and N. Mavalvala, presented at the Gordon Research Conference on Mechanical Systems in the Quantum Regime, Galveston, TX (2010).

[94] C. M. Mow-Lowry, A. J. Mullavey, S. Gossler, M. B. Gray, and D. E. McClelland, Phys. Rev. Lett. 100, 010801 (2008). 